\title{
Super Beltrami Differentials
}

\author{
Michiaki Takama \\ Research Institute for Mathematical Sciences, Kyoto University, Kyoto, 606 Japan
}

Received March 12, 1991

\begin{abstract}
Superconformal structures on a given 2|2-dim supermanifold give rise to the notion of super Riemann surfaces (SRS's). We investigate super Beltrami coefficients which parametrize (almost) superconformal structures on the supermanifold. The integrability condition of the structure reduces to a simple relation among the coefficients. Taking this into account, we can write the super Beltrami equations in a transparent form. Then an analysis of these equations enables us to prove the possibility of the special gauge choice of the Wess-Zumino type for the super Beltrami differentials. This gauge choice simplifies the description of the deformations of SRS's considerably and its existence will afford a better understanding of the structure of the super Teichmüller space.
\end{abstract}

\section{Introduction}

Two-dimensional field theories perceive the space-time which they inhabit to be a super Riemann surface (SRS) if their couplings to supergravity are superconformally invariant. The theory of SRS's plays an important role in the studies of the two-dimensional supergravity, superstring in the Neveu-Schwarz formalism and superconformal field theories. For example, holomorphy properties of the superstring measure on the moduli space of SRS's (super moduli space) are the fundamental importance of the theory [1].

There are mainly two ways in defining SRS's, that is, patch definition [2] (including algebraic definition [3]) and frame definition [4, 5], and for each of them there exist corresponding approaches to the moduli problem [3-6]. In this paper, we develop the study of the super Beltrami equations, clarifying the basic properties of the super Beltrami differentials and investigate the Teichmüller deformations of SRS's of genus $p>1$.

This approach is based on the frame definition of SRS's in which the notion of SRS is derived from 2-d supergravity geometry. In this respect, it is fit for the direct applications to physics [7-9]. The geometrical structure on 2/2-supermanifolds 
suitable for 2-d supergravity is established by Howe [10]. He set up torsion constraints properly, assuming the reduction of the structure group from $G L(2 \mid 2, \mathbb{R})$ to $U(1)$ (local Lorentz group), and defined the super Weyl transformations of the frame fields. The geometrical meanings of these constraints were clarified by Giddings and Nelson [5]. They showed that the constraints of [10] are basically the integrability conditions of the reduction of the structure group to a certain subgroup $G$. (The difference as gauge groups between $U(1) \times$ [super Weyl] and $G$ has only secondary meaning.) A distribution (a subbundle of the tangent bundle) that provides a reduction concerned can be defined through Pfaff equations. The three coefficients of the Pfaffian form (omitting the scale factor) form a set of super Beltrami coefficients $(\mu, \varrho, v)$. Here $\varrho$ and $v$ are Grassmann odd superconformal tensors of weight $\left(-1, \frac{1}{2}\right)$ and $\left(-\frac{1}{2}, 0\right)$ respectively, while $\mu$ is a even superfield and not a pure superconformal tensor.

The super-extension of the Teichmüller theory was first studied by Crane and Rabin [11]. They represent an SRS on the universal covering space, taking a quotient by the action of a discrete subgroup of the super Möbius transformations. They derived the super Beltrami equations, discussed their solution and studied the extension of the Bers' embedding theorem to exhibit the complex structure of the super Teichmüller space. However, their treatment of the super Beltrami coefficients included insufficient points. To describe the deformations of SRS's, they took a split SRS as the deformation origin and made a pregauge-fixing for the super Beltrami coefficients of the form $\sigma \equiv \varrho+\bar{\theta} \mu=0$, conjecturing that it was always possible. Thus the structure of the space of the coefficients and how they contribute to the deformations have not been clarified in the full context. Though much work has been made on the super Teichmüller theory [12-16], there still remain things even in the elementary ground of the theory.

In the present paper, we give the complete treatment of the super Beltrami equations that has not been presented so far and solve the problem of the gauge choice of $[11,12]$. The progress starts with the following remark. The integrability condition for the super Beltrami coefficients is simply that $\mu$ is expressed as a certain function of $\varrho, v$ and their derivatives. ${ }^{1}$ (Regarding this point, the approach through the Pfaffian form seems much simpler than the vector field approach studied in [15], in which the integrability condition leads to complicated equations among the coefficients of the vector fields.) So, among three coefficients, two odd ones $\varrho$ and $v$ are considered as the basic fields [13], which we call super Beltrami differentials. The even superfield $\mu$ can be viewed as the super-extension of the ordinary Beltrami differential. An interesting point is that the super Beltrami coefficients appear in the essential parts of the super Beltrami equations only through the (modified) even coefficient $\tilde{\mu}$ in which the basic odd differentials are nested. This observation enables us to prove the conjecture for the gauge choice of [11] in a stronger form. It states that, taking a split SRS as the deformation origin, whole deformations of SRS's are sufficiently covered by considering the super Beltrami differentials of special form such that $v=0$ and $\left(1-\overline{\theta \partial}_{\theta}\right) \varrho=0$. This gauge condition, which is imposed uniformly on the universal covering space, is a kind of gauge choice usually employed in the physics literature as Wess-Zumino gauge. Its validity has been shown for the infinitesimal deformations [12,13], (although

\footnotetext{
${ }^{1}$ When struggling to complete the paper, I found that the constraints among the super Beltrami coefficients, which express the integrability condition, had been already solved by a somewhat different manner in [8], though the result itself I obtained a year ago. In [8], the authors are mainly intent on the physical application to some superconformal models. The super Beltrami equations are not investigated there
} 
negative arguments were made for arbitrary deformations in [12]). This gauge choice simplifies the description of the deformations of SRS considerably and its existence will improve our understandings of the structure of the super Teichmüller space.

The article is organized as follows. In Sect. 1, we describe superconformal structures in terms of "metric" from which the Pfaffian forms are derived. In Sect. 2, we derive the integrability condition and discuss the basic properties of the super Beltrami differentials. In Sect. 3, the super Beltrami equations are investigated in detail in terms of the components of the superfields. We prove the possibility of the special gauge choice of the Wess-Zumino type in Sect. 4. We see that one of the super Beltrami differentials $v$ of weight $\left(-\frac{1}{2}, 0\right)$ plays a role of the background spin field which changes the spin structures of the deformed SRS's. In Appendix A, we summarize the infinitesimal deformations, attending to the problem of the gauge choice when a general (non-)split SRS is taken as the deformation origin. Under a plausible assumption about the boundary behavior of the super Beltrami differentials, it can be shown that the $v=0$ gauge is always possible. In Appendix B, residual symmetry under the Wess-Zumino gauge is discussed. Appendix $C$ is devoted to a brief description of the supercomplex structure of the super Teichmüller space through the infinitesimal approach. The Wess-Zumino gauge supplies a class of natural complex coordinate systems for the super Teichmüller space.

Throughout this paper, we do not enter into details of the specific constructions of supermanifolds. ${ }^{2}$ We always denote the body map by $\pi$.

\section{Superconformal Structure}

An almost superconformal structure of a real 2|2-dimensional supermanifold $M$ is a reduction of the structure group [5]:

$$
G L(2 \mid 2, \mathbb{R}) \rightarrow G=\left\{\left(\begin{array}{cc}
A^{2} & * \\
0 & A
\end{array}\right) \in G L(1 \mid 1, \mathbb{C})\right\} .
$$

When the almost superconformal structure is integrable, it is called superconformal structure. Here we describe this structure in terms of "metric" on $M$.

Definition 1.1. A metric $g$ on $M$ is a degenerate symmetric two-form with the following properties.

(i) $\pi(g)$ defines an ordinary metric on $\pi(M)$.

(ii) At every point $x$ of $M$, any Grassmann odd vector field $v$ in a neighborhood of $x$ with vanishing norm at $x$ [i.e. $g_{x}\left(v_{x}, v_{x}\right)=0$ ] satisfies $\{v, v\} \neq 0$, and such $v$ spans at $x 0 \mid 2$-dimensional subspace $\mathscr{D}_{x}$ of $T_{x} M$.

By (i) and (ii) $g$ defines a distribution $\mathscr{D}$ on $M$ together with an orthogonal structure, and has a local expression

$$
g=\left|E^{z}\right|^{2} .
$$

Then $\operatorname{Re} E^{z}$ and $\operatorname{Im} E^{z}$ give the Pfaffian forms defining the distribution $\mathscr{D}$. It is possible to choose the local basis of $\mathscr{D}$ denoted by $E_{1}$ and $E_{2}$ such that

$$
\begin{gathered}
g\left(\left(E_{1}\right)^{2}-\left(E_{2}\right)^{2},\left(E_{1}\right)^{2}-\left(E_{2}\right)^{2}\right)=g\left(\left\{E_{1}, E_{2}\right\},\left\{E_{1}, E_{2}\right\}\right)=1, \\
g\left(\left(E_{1}\right)^{2}-\left(E_{2}\right)^{2},\left\{E_{1}, E_{2}\right\}\right)=0 .
\end{gathered}
$$

${ }^{2}$ For various approaches to define supermanifolds, see [17] and also [18] 
Then $E_{1}$ and $E_{2}$ are determined up to $O(2)$ rotation. With these $E_{1}$ and $E_{2}$, we can define a complete frame basis by $\left\{E_{1}, E_{2},\left(E_{1}\right)^{2}-\left(E_{2}\right)^{2},\left\{E_{1}, E_{2}\right\}\right\}$. Consider the complexification of the tangent $T^{c} M$ and set $E_{+}=E_{1}+i E_{2},{ }^{3}$ then

$$
\left(E_{+}\right)^{2}=\left(E_{1}\right)^{2}-\left(E_{2}\right)^{2}+i\left\{E_{1}, E_{2}\right\}
$$

If $E_{1}, E_{2}$ are related to $\widetilde{E}_{1}, \widetilde{E}_{2}$ [which satisfy (1.2)] through a $O(2)$ rotation in some neighborhood:

$$
\tilde{E}_{+}=e^{i \varphi} E_{+},
$$

then it follows that

$$
\left(\tilde{E}_{+}\right)^{2}=e^{2 i \varphi}\left(E_{+}\right)^{2}+i 2\left(E_{+} \varphi\right) e^{2 i \varphi} E_{+} .
$$

Therefore $\left(\widetilde{E}_{+}\right)^{2}, \widetilde{E}_{+}$and $\left(E_{+}\right)^{2}, E_{+}$differ by an element of $G$ with $A=e^{i \varphi}$ in (1.1).

Similarly to the ordinary case, we call the metrics $g$ and $g^{\prime}$ on $M$ conformally equivalent if and only if $g$ and $g^{\prime}$ are proportional at every point of $M$. Let $\mathscr{F}(M)$ denote the set of all almost superconformal structures on $M$. From the description made above, it is clear that there is one to one correspondence between the conformal classes of metrics on $M$ and $\mathscr{F}(M)$. According to the notations in [11], we write $\left|E^{z}\right|$ as

$$
\begin{aligned}
\left|E^{z}\right| & =e^{\Phi}|d z+\mu d \bar{z}+\hat{v} d \theta+\sigma d \bar{\theta}| \\
& =e^{\Phi}\left|e^{z}+\mu e^{\bar{z}}+v d \theta+\varrho d \bar{\theta}\right|,
\end{aligned}
$$

where

$$
\begin{gathered}
e^{z}=d z+\theta d \theta, \quad e^{\bar{z}}=\overline{e^{z}}=d \bar{z}+\bar{\theta} d \bar{\theta}, \\
\hat{v}=v+\theta, \quad \varrho=\sigma-\bar{\theta} \mu .
\end{gathered}
$$

Super Beltrami coefficients $\mu, v$, and $\varrho$ are complex valued superfields, the former with Grassmann even parity, while the latter two with Grassmann odd parities.

Integrable almost superconformal structure, say superconformal structure means that there exist local coordinate systems $(u, \phi)$ in which $E^{z}$ is proportional to $d u+\phi d \phi$. As we will see in the next section, the integrability condition is reduced to a certain constraint among the super Beltrami coefficients $\mu, v$, and $\varrho$, from which $\mu$ (and hence $\sigma$ ) can be expressed in terms of $\varrho$ and $\nu$. For a given superconformal structure, the natural coordinate systems $(u, \phi)$ are found by solving the super Beltrami equations $[11]^{4}$ :

$$
\begin{gathered}
w:(z, \theta) \mapsto(u, \phi)=\left(w_{b}(z, \theta), w_{f}(z, \theta)\right), \\
\mu=\frac{\bar{\partial}_{z} w_{b}+w_{f} \bar{\partial}_{z} w_{f}}{\partial_{z} w_{b}+w_{f} \partial_{z} w_{f}}, \\
\sigma=\frac{-\bar{\partial}_{\theta} w_{b}+w_{f} \bar{\partial}_{\theta} w_{f}}{\partial_{z} w_{b}+w_{f} \partial_{z} w_{f}}, \\
D_{v} w_{b}=w_{f} D_{v} w_{f},
\end{gathered}
$$

3 We have a direct sum decomposition of $\mathscr{D}^{c}: \mathscr{D}^{c}=\mathscr{D}_{+} \oplus \mathscr{D}_{-}$, where $\mathscr{D}_{+}\left(\mathscr{D}_{-}\right)$is spanned by such $E_{+}\left(E_{-} \equiv \overline{E_{+}}\right)$'s

${ }^{4}$ In the following we will write superdiffeomorphisms as $w=\left(w_{b}, w_{f}\right)$, where $w_{b}, w_{f}$ imply the bosonic part and the fermionic part of the map $w$, respectively 
where

$$
D_{v}=\partial_{\theta}+\hat{v} \partial_{z}=D+v \partial_{z} .
$$

The supermanifold $M$ with such coordinate systems becomes a super Riemann surface (SRS), i.e. 1|1-dimensional supercomplex manifold patched together with superconformal transformations [2]. Conversely every SRS has a metric locally proportional to $|d z+\theta d \theta|^{2}$ [14], and hence possesses a natural superconformal structure. In this way a real 2/2-dimensional supermanifold with a given superconformal structure is viewed as an SRS. With this fact, we can.proceed in the analogous steps to the ordinary Teichmüller theory. ${ }^{5}$

We fix some standard SRS $\Sigma$ such that its body is a marked Riemann surface of genus $p$ (provided with a certain spin structure.) If $\Sigma^{\prime}$ is another SRS of genus $p$, we can find a superdiffeomorphism $w: \Sigma \rightarrow \Sigma^{\prime}$ (since $\Sigma$ and $\Sigma^{\prime}$ are equivalent as smooth supermanifold [21]). We choose a metric $g^{\prime}$ on $\Sigma^{\prime}$ belonging to its natural superconformal structure and pull back the metric $g^{\prime}$ by the map $w$ to $g=w^{*} g^{\prime}$ on $\Sigma$. Then the metric $g$ carries a superconformal structure on $\Sigma$, defining the SRS equivalent to $\Sigma^{\prime}$. Let $\mathscr{M}(\Sigma)$ denote the set of all superconformal structures on $\Sigma$. We see that every SRS of genus $p$ is equivalent to a some superconformal structure on $\Sigma$. However, since there are many possible such superdiffeomorphisms from $\Sigma$ to $\Sigma^{\prime}$, an identical SRS is represented by many superconformal structures on $\Sigma$. This leads us to define an equivalence relation in $\mathscr{M}(\Sigma)$. Let $s \operatorname{Diff}^{+}(\Sigma)$ be the group of sense preserving ${ }^{6}$ superdiffeomorphisms of $\Sigma$ and $\operatorname{sDiff}_{0}(\Sigma)$ be the subgroup of $s \operatorname{Diff}^{+}(\Sigma)$ which consist of the elements homotopic to the identity map. Then ${ }_{s \operatorname{Diff}}{ }^{+}(\Sigma)$ acts on $\mathscr{M}(\Sigma)$ via the pullback. Two superconformal structures $\Psi=(\varrho, v)$ and $\Psi^{\prime}=\left(\varrho^{\prime}, v^{\prime}\right)$ are Teichmüller equivalent $\Psi \sim \Psi^{\prime}$ if and only if there exists a map $w \in s \operatorname{Diff}_{0}(\Sigma)$ such that $\Psi=w^{*} \Psi^{\prime}$. The super Teichmüller space $\mathrm{ST}_{p}(\Sigma)$ of SRS's of genus $p$ is the quotient space $\mathscr{M}(\Sigma) / s \operatorname{Diff}_{0}(\Sigma)$.

\section{Super Beltrami Differentials and the Integrability Condition}

In Sect. 1, we viewed the super Teichmüller space $\mathrm{ST}_{p}$ in terms of the superconformal structures on a given SRS $\Sigma$. To obtain a concrete description for $\mathrm{ST}_{p}$, we adopt the uniformization theorem for SRS's as usual [11]. In the following, we restrict our studies to SRS's of genus $p \geqq 2$. Let SH be the super upper half-plane, i.e.

$$
\mathrm{SH}=\{(z, \theta) \mid \operatorname{Im} \pi(z)>0\} .
$$

Every compact SRS of genus $p \geqq 2$ is realized as a quotient $\mathrm{SH} / \Gamma$, where

(i) $\Gamma$ is a subgroup of Aut $(\mathrm{SH})$ (the group of all superconformal automorphisms of $\mathrm{SH}$ ) isomorphic to the standard surface group $\Gamma_{p}$, which is a group generated by elements $a_{1}, \ldots, a_{2 g}$ with a relation

$$
a_{1} a_{2} a_{1}^{-1} a_{2}^{-1} \ldots a_{2 g-1} a_{2 g} a_{2 g-1}^{-1} a_{2 g}^{-1}=1 .
$$

(ii) $\pi(\Gamma)$ is an ordinary Fuchsian group which represents the underlining Riemann surface $\pi(\Sigma)$.

\footnotetext{
${ }^{5}$ For introductions to Teichmüller theory and the theory of quasiconformal mappings, see, for example $[19,20]$

6 By the word "sense preserving," we mean that both the super Jacobian and the Jacobian of the body map are positive
} 
Let $\vartheta$ be the isomorphism from $\Gamma_{p}$ into Aut(SH) such that $\vartheta\left(\Gamma_{p}\right)=\Gamma$. Evidently, two such groups $\Gamma_{1}$ and $\Gamma_{2}$ represent an equivalent SRS if and only if they are related by the conjugation $\Gamma_{1}=A \Gamma_{2} A^{-1}, A \in \operatorname{Aut}(\mathrm{SH})$. As pointed out in [11], Aut $(\mathrm{SH})$ is a much larger group than the group of all linear transformations of $\mathrm{SH}^{7}$ However, in [14], Hodgkin showed that for a given $\Gamma$, there exists a superconformal map $F$ [which is unique up to the conjugation by elements in $\operatorname{SPL}(2, \mathbb{R})]$ such that $F \Gamma F^{-1}$ is contained in $\operatorname{SPL}(2, \mathbb{R})$. By this fact, we can replace $\operatorname{Aut}(\mathbf{S H})$ with $\operatorname{SPL}(2, \mathbb{R})$ in the condition (i) for the "super Fuchsian group" presented above. Thus it is sufficient to consider isomorphisms $\vartheta$ from $\Gamma_{p}$ into $\operatorname{SPL}(2, \mathbb{R})$ with the equivalence relation under oberall $\operatorname{SPL}(2, \mathbb{R})$ conjugations. To fix the freedoms of the $\operatorname{SPL}(2, \mathbb{R})$ conjugations, we normalize $\vartheta$ such that the fixed points of $\vartheta\left(a_{1}\right)=\left(\vartheta_{b}\left(a_{1}\right), \vartheta_{f}\left(a_{1}\right)\right)$ are $(0,0),(\infty, 0)$ and the even coordinate of the attractive fixed point of $\vartheta\left(a_{2}\right)$ is specified to be 1 , i.e. $\vartheta_{b}\left(a_{2}\right)(1,0)=1$. After imposing this condition, it remains one freedom of the conjugation by the fermionic inversion $I:(z, \theta) \rightarrow(z,-\theta)[11] . \Gamma$ and $I \Gamma I$ are different only in the signs of the odd parameters. This overall sign ambiguity of the odd parameters of $\Gamma$ results in the super-orbifold structure of the $\mathrm{ST}_{p}[11]$, which we will see in the later sections.

On a given $\mathrm{SRS} \Sigma=\mathrm{SH} / \Gamma$, let us put an almost superconformal structure

$$
(\mu, \varrho, v) \in \mathscr{F}(\mathrm{SH} / \Gamma)
$$

as in (1.3). This structure lifts to an almost superconformal structure on SH. Then the lifted super Beltrami coefficients transform with respect to the cover transformation $A \in \Gamma$ as follows: ${ }^{8}$

$$
\begin{aligned}
& \varrho \circ A=\frac{\left(D A_{f}\right)\left(D_{v} A_{f}\right)}{\overline{D A}_{f}} \varrho \\
& v \circ A=\left(D A_{f}\right) v, \\
& \mu \circ A=\frac{\left(D A_{f}\right)\left(D_{v} A_{f}\right)}{\overline{D A}_{f}^{2}}\left(\mu+\frac{\overline{\partial_{z} A_{f}}}{\overline{D A_{f}}} \varrho\right),
\end{aligned}
$$

where $A(z, \theta)=\left(A_{b}(z, \theta), A_{f}(z, \theta)\right)$.

In (2.1), we notice that $D_{v} A_{f}$ partly appears instead of $D A_{f}$ in the transition functions of $\varrho$ and $\mu$. However it is no matter. Because of

$$
\left(D_{v} \hat{v}\right) \circ A=\frac{D A_{f}}{D_{v} A_{f}} D_{v} \hat{v},
$$

we can reform them to have the normal transition functions by multiplying $D_{v} \hat{v}$ if necessary. From (2.1), $\varrho$ and $v$ are superconformal tensors of weight $\left(-1, \frac{1}{2}\right),\left(-\frac{1}{2}, 0\right)$ respectively, while $\mu$ is of weight $(-1,1)$ but not a pure superconformal tensor.

\footnotetext{
$7 \operatorname{SPL}(2, \mathbb{R})$ is the subgroup of $\operatorname{SPL}(2, \mathbb{C})$ consisting of the elements which fix the "super real line" $S \mathbb{R}=\{(z, \theta) \mid z=\bar{z}, \theta=\bar{\theta}\}$. The boundary of $\mathrm{SH}$, which we denote by $\partial \mathrm{SH}$, is not an honest super manifold and differs from $S \mathbb{R}$. Note that $\operatorname{SPL}(2, \mathbb{C})$ is isomorphic to Aut $(S \hat{\mathbb{C}})$ that is the group of all automorphisms of the supersphere $S \hat{\mathbb{C}}$

${ }^{8}$ Here and after we use the same notation for the lifted super Beltrami coefficients
} 
Proposition 2.1. The super Beltrami equations (1.4) is rewritten as

$$
\begin{gathered}
\bar{D} w_{b}=w_{f} \bar{D} w_{f}-\frac{\left(D_{v} w_{f}\right)^{2}}{D_{v} \hat{v}} \varrho, \\
D_{\nu} w_{b}=w_{f} D_{\nu} w_{f}, \\
\mu=-\bar{D}_{\varrho}+\frac{1}{4}\left(D_{v} \hat{v}\right)\{G(\varrho, v)\}^{2}+\partial_{z} \varrho \varrho,
\end{gathered}
$$

where

$$
G(\varrho, v)=D_{v}\left[\frac{\varrho}{D_{v} \hat{v}}\right]+\frac{\bar{D} v}{D_{v} \hat{v}}
$$

Proof. From (1.4c) and using the fact $D_{v}^{2}=\left(D_{v} \hat{v}\right) \partial_{z}$, we get $\partial_{z} w_{b}+w_{f} \partial_{z} w_{f}=\frac{\left(D_{v} w_{f}\right)^{2}}{D_{v} \hat{v}}$. (Note that $D_{v} \hat{v}$ must be invertible.) Thus (2.2a) is obtained from (1.4a) and (1.4b) immediately. Differentiating $(2.2 \mathrm{a})$ with $\bar{D}$, we have

$$
\frac{D_{v} \hat{v}}{\left(D_{v} w_{f}\right)^{2}}\left\{\left(\bar{D} w_{f}\right)^{2}-\bar{D}\left[\frac{\left(D_{v} w_{f}\right)^{2}}{D_{v} \hat{v}} \varrho\right]\right\}=\frac{\bar{\partial}_{z} w_{b}+w_{f} \bar{\partial}_{z} w_{f}}{\partial_{z} w_{b}+w_{f} \partial_{z} w_{f}}=\mu .
$$

Since $\left\{\bar{D}, D_{v}\right\}=(\bar{D} v) \partial_{z}$, the compatibility of (2.2) leads the following equation which involves $w_{f}$ only:

$$
\bar{D} w_{f}=-\varrho \partial_{z} w_{f}+\frac{1}{2} G(\varrho, v) D_{v} w_{f} .
$$

By putting this equation into the left-hand side of the above expression of $\mu$, we obtain the formula (2.3). Conversely, if $(\mu, \varrho, v)$ satisfies $(2.3),(1.4 \mathrm{a})$ is obtained from (2.2). Then (2.2a) and $\varrho=\sigma-\bar{\theta} \mu$ imply $(1.4 \mathrm{~b})$. Finally we note that $(2.3)$ is consistent with the transformation properties given by (2.1). Q.E.D.

The proposition states that the solutions of the super Beltrami equations (1.4) are controlled only through $\varrho$ and $v$. In the following, by "super Beltrami equations" we mean (2.2). As we have already seen, the compatibility of (2.2) gives the equation for $w_{f}$. Moreover it leads to a chain of the equations.

Corollary 2.2. The solution $w=\left(w_{b}, w_{f}\right)$ of the super Beltrami equations satisfies a pair of equations

$$
\begin{aligned}
& \bar{D} w_{f}=-\varrho \partial_{z} w_{f}+\frac{1}{2} G(\varrho, v) D_{v} w_{f}, \\
& \bar{D} w_{b}=-\varrho \partial_{z} w_{b}+\frac{1}{2} G(\varrho, v) D_{v} w_{b}
\end{aligned}
$$

and also

$$
\begin{aligned}
& \bar{\partial}_{z} w_{f}=\mu \partial_{z} w_{f}+\frac{1}{2} \eta(\mu, v) D_{v} w_{f}, \\
& \bar{\partial}_{z} w_{b}=\mu \partial_{z} w_{b}+\frac{1}{2} \eta(\mu, v) D_{v} w_{b},
\end{aligned}
$$

where $\eta(\mu, v)$ can be written as

$$
\eta(\mu, v)=D_{v}\left[\frac{\mu}{D_{v} \hat{v}}\right]+\frac{\bar{\partial}_{z} v}{D_{v} \hat{v}}
$$

Proof. Putting (2.5a) into (2.2a) and using (2.2b), we have Eq. (2.5b). Equations (2.6) are obtained by differentiating $(2.5)$ by $\bar{D}$. Then, employing the formula (2.3), the direct computation shows that the expression for $\eta$ is given by (2.7). Q.E.D. 
Remark 2.3. The superconformal transforms of the solution of (2.2) are also the solutions of the same Eq. (2.2).

As pointed out in [11], the existence of the solution of Eqs. (2.2) is ensured. We complete their discussion in the next section.

To guarantee the solution to be a sense preserving superdiffeomorphism (which we call quasisuperconformal map), ${ }^{9}$ we must impose certain bounds on $(\varrho, v)$ as in the ordinary case. Taking account of the properties of the superdeterminant and the super Beltrami equations (1.4),(2.2),(2.5), and (2.6), we can express the Jacobian of the transformation $w:(z, \theta) \rightarrow\left(w_{b}, w_{f}\right)$ in terms of super Beltrami differentials,

$$
\begin{gathered}
J=\operatorname{sdet}\left[\begin{array}{llll}
\partial_{z} w_{b} & \partial_{z} \bar{w}_{b} & \partial_{z} w_{f} & \partial_{z} \bar{w}_{f} \\
\partial_{z} w_{b} & \bar{\partial}_{z} \bar{w}_{b} & \bar{\partial}_{z} w_{f} & \bar{\partial}_{z} \bar{w}_{f} \\
\partial_{\theta} w_{b} & \partial_{\theta} \bar{w}_{b} & \partial_{\theta} w_{f} & \partial_{\theta} \bar{w}_{f} \\
\bar{\partial}_{\theta} w_{b} & \bar{\partial}_{\theta} \bar{w}_{b} & \bar{\partial}_{\theta} w_{f} & \bar{\partial}_{\theta} \bar{w}_{f}
\end{array}\right] \\
=\operatorname{sdet}\left[\begin{array}{llll}
\partial_{z} w_{b}+w_{f} \partial_{z} w_{f} & \partial_{z} \bar{w}_{b}+\bar{w}_{f} \partial_{z} \bar{w}_{f} & \partial_{z} w_{f} & \partial_{z} \bar{w}_{f} \\
\bar{\partial}_{z} w_{b}+w_{f} \bar{\partial}_{z} w_{f} & \bar{\partial}_{z} \bar{w}_{b}+\bar{w}_{f} \bar{\partial}_{z} \bar{w}_{f} & \bar{\partial}_{z} w_{f} & \bar{\partial}_{z} \bar{w}_{f} \\
D w_{b}-w_{f} D w_{f} & D \bar{w}_{b}-\bar{w}_{f} D \bar{w}_{f} & D w_{f} & D \bar{w}_{f} \\
\bar{D} w_{b}-w_{f} \bar{D} w_{f} & \bar{D} \bar{w}_{b}-\bar{w}_{f} \bar{D} \bar{w}_{f} & \bar{D} w_{f} & \bar{D} \bar{w}_{f}
\end{array}\right] \\
=\frac{\left|D_{v} w_{f}\right|^{2}}{\left|D_{v} \hat{v}\right|} \operatorname{sdet}\left[\begin{array}{cccc}
1 & \bar{\mu} & 0 & \frac{1}{2} \bar{\eta} \\
\mu & 1 & \frac{1}{2} \eta & 0 \\
-v & -\bar{\varrho} & 1 & \frac{1}{2} \bar{G} \\
-\varrho & -\bar{v} & \frac{1}{2} G & 1
\end{array}\right] .
\end{gathered}
$$

Since $\pi(J)$ should be positive, we obtain

$$
\pi\left(\frac{1-|\mu|^{2}}{1-\frac{1}{4}|G|^{2}}\right)>0 \text {. }
$$

As it is easily seen from (2.6) that $\pi\left(1-|\mu|^{2}\right)>0$ is the condition for the body map of $w$ to be sense preserving, we have the following boundedness conditions:

$$
\begin{gathered}
D_{v} \hat{v} \text { must be invertible, } \\
|\pi(\mu(\varrho, v))|<1, \\
|\pi(G(\varrho, v))|<2 .
\end{gathered}
$$

Note that $|\pi(\mu)|,|\pi(G)|$ are independent of the choice of the local coordinates. The super Beltrami differentials $(\varrho, v)$ should be bounded through the formulas $\mu$ and $G$ in a somewhat complicated way. The condition (2.9a) needs more explorations. The following observation is indicated in [15], and we will show it explicitly in Sect. 4. When we consider the condition (2.9) on a SRS $\Sigma,(2.9 \mathrm{a})$ means that $\pi\left(D_{v} \hat{v}\right)$ $=1+\pi\left(\partial_{\theta} v\right)$ is a map from $\pi(\Sigma)$ to $\mathbb{C}-\{0\}$. The homotopy classes of such a maps are classified by the elements of $\mathbb{Z}^{2 p}$ which count the twists around the handles of

9 We have no intention to be completely rigorous. Throughout this paper we assume that all functions $(\varrho, v)$ are smooth and as a consequence the map $w$ becomes smooth 
$\pi(\Sigma)$. A solution of Eqs. (2.2) defines a superdiffeomorphism from $\Sigma$ to some SRS $\Sigma^{\prime}$ and send the marking of $\Sigma$ to that of $\Sigma^{\prime}$. Then the spin structure of $\Sigma^{\prime}$ with respect to this marking changes according to the homotopy class of $\pi\left(D_{v} \hat{v}\right)$ modulo $(2 \mathbb{Z})^{2 p}$. Thus as long as the deformations of SRS's with the same spin structure, we should replace the condition of (2.9a) with more restricted one:

$$
1+\pi\left(\partial_{\theta} v\right) \text { is nullhomotopic as a map from } \pi(\Sigma) \text { to } \mathbb{C}-\{0\} .
$$

For a given $(\mu, \varrho, v) \in \mathscr{F}(\mathrm{SH} / \Gamma),(2.3)$ is the integrability condition, and hence the superconformal structure is completely specified by $(\varrho, v)$, which allows us to write as $(\varrho, v) \in \mathscr{M}(\mathrm{SH} / \Gamma)$. Let us denote by $\mathrm{SB}(\Gamma)$ the linear space of the super Beltrami differentials on $\mathrm{SH}$ which satisfy $(2.1 \mathrm{a}, \mathrm{b})$, and by $\mathrm{SB}(\Gamma)_{b}$ the space of those bounded by the conditions $(2.9 \mathrm{~b}, \mathrm{c})$ and $\left(2.9 \mathrm{a}^{\prime}\right)$. Then we identify $\mathscr{M}(\mathrm{SH} / \Gamma)$ with $\mathrm{SB}(\Gamma)_{b}$.

Next we investigate the composition law for the super Beltrami differentials which plays important role in (super) Teichmüller theory. Let $\Sigma_{1}$ and $\Sigma_{2}$ be SRS's and $w: \Sigma_{1} \rightarrow \Sigma_{2}$ be a quasisuperconformal map. As was mentioned before, through the pullback of the natural superconformal structure of $\Sigma_{2}, w$ induces a superconformal structure on $\Sigma_{1}$ that is given by the formulas (1.4). We denote it by $\Psi_{w}=\left(\varrho_{w}, v_{w}\right)$. The expression of $\Psi_{w}$ does not depend on the choice of the local coordinates of $\Sigma_{2}$.

Proposition 2.4. Let $h: \Sigma_{0} \rightarrow \Sigma_{1}, g: \Sigma_{0} \rightarrow \Sigma_{2}$, and $w=g \circ h^{-1}: \Sigma_{1} \rightarrow \Sigma_{2}$ be quasisuperconformal maps among SRS's $\Sigma_{0}, \Sigma_{1}$, and $\Sigma_{2}$, and write the induced super Beltrami differentials as $\Psi_{h}, \Psi_{g}$, and $\Psi_{w}$. Then

$$
\begin{aligned}
& \left(\mu_{g}-\mu_{h}, \varrho_{g}-\varrho_{h}, v_{g}-v_{h}\right) H=\left(\mu_{w}, \varrho_{w}, v_{w}\right) \circ h \\
& \quad \times\left[\begin{array}{ccc}
\left(1-\mu_{g} \bar{\mu}_{h}\right) \bar{H} & \left(\bar{v}_{h}-\varrho_{g} \bar{\mu}_{h}\right) \bar{H} & \left(\bar{\varrho}_{h}-v_{g} \bar{\mu}_{h}\right) \bar{H} \\
\left(\bar{\partial}_{z}-\mu_{g} \partial_{z}\right) \overline{h_{f}} & \left(\bar{D}+\varrho_{g} \partial_{z}\right) \overline{h_{f}} & D_{v_{g}} \bar{h}_{f} \\
\left(\bar{\partial}_{z}-\mu_{g} \partial_{z}\right) h_{f} & \left(\bar{D}+\varrho_{g} \partial_{z}\right) h_{f} & D_{v_{g}} h_{f}
\end{array}\right],
\end{aligned}
$$

where $\mu$ 's are defined by the formula (2.3) and $H \equiv \partial_{z} h_{b}+h_{f} \partial_{z} h_{f}=\frac{\left(D_{v_{h}} h_{f}\right)^{2}}{D_{v_{h}} \hat{v}_{h}}$.

Proof. It can be derived from the chain rules for differentiations. ${ }^{10}$ Q.E.D.

Note that there are no appearances of the complex conjugations of $\varrho_{g}, v_{g}, \mu_{g}$ in (2.10) and that $\mu$ depends holomorphically on $\varrho$ and $v$ in (2.3), which become the crucial point for the complex structure of the super Teichmüller space. From Proposition 2.4, we also remark that the superdiffeomorphism group sDiff ${ }^{+}(\mathrm{SH})$ has a representation on $\mathscr{M}(\mathrm{SH})$, on setting

$$
\Psi_{w} * \Psi_{h}=\Psi_{g}
$$

${ }^{10}$ For any superfunction $F(z, \bar{z}, \theta, \bar{\theta})$,

$$
\begin{aligned}
D[F \circ h]= & \left(D h_{f}\right)\{(D F) \circ h\}+\left(D h_{b}-h_{f} D h_{f}\right)\left\{\left(\partial_{z} F\right) \circ h\right\} \\
& +\left(D \overline{h_{f}}\right)\{(\bar{D} F) \circ h\}+\left(D \overline{h_{b}}-\overline{h_{f}} D \overline{h_{f}}\right)\left\{\left(\bar{\partial}_{z} F\right) \circ h\right\}, \\
\partial_{z}[F \circ h]= & \left(\partial_{z} h_{f}\right)\{(D F) \circ h\}+\left(\partial_{z} h_{b}+h_{f} \partial_{z} h_{f}\right)\left\{\left(\partial_{z} F\right) \circ h\right\} \\
& +\left(\partial_{z} \overline{h_{f}}\right)\{(\bar{D} F) \circ h\}+\left(\partial_{z} \overline{h_{b}}+\overline{h_{f}} \partial_{z} \overline{h_{f}}\right)\left\{\left(\bar{\partial}_{z} F\right) \circ h\right\} .
\end{aligned}
$$




\section{Super Beltrami Equations}

In this section, we investigate the super Beltrami equations (2.2) in detail, discuss their solutions and describe the deformations of the super Fuchsian groups by making use of them. To begin with, we consider Eqs. (2.2) on the superplane $S \mathbb{C}$. Let $\Psi \in \mathscr{M}(S \mathbb{C})$, assuming that $\varrho$ and $v$ are smooth superfunctions on $S \mathbb{C}$. For any superfunction $F(z, \bar{z}, \theta, \bar{\theta})$, it is convenient to separate it into the $\bar{\theta}$-free part and the $\bar{\theta}$-containing part, which we write as $F^{P}$ and $F^{Q}$, respectively:

$$
F^{P}=\left(1-\overline{\theta \partial}_{\theta}\right) F, \quad F^{Q}=\bar{\partial}_{\theta} F .
$$

Note that $\bar{D} F=F^{Q}+{\overline{\theta \partial_{z}}}_{z} F^{P}$. We expand the super Beltrami differentials in terms of $\theta, \bar{\theta}$ :

$$
\begin{gathered}
\varrho=\varrho^{P}+\bar{\theta} \varrho^{Q}, \quad \varrho^{P}=\varrho_{1}+\theta \varrho_{0}, \quad \varrho^{Q}=\varrho_{2}-\theta \varrho_{3}, \\
v=v^{P}+\bar{\theta} v^{Q}, \quad v^{P}=v_{1}+\theta v_{0}, \quad v^{Q}=v_{2}-\theta v_{3}, \\
\mu(\varrho, v)=\mu^{P}+\bar{\theta} \mu^{Q}, \quad \mu^{P}=\mu_{0}+\theta \mu_{1} .
\end{gathered}
$$

We also expand the functions $w_{b}$ and $w_{f}$ :

$$
\begin{aligned}
& w_{b}=w_{b}^{P}+\bar{\theta} w_{b}^{Q}=w_{b \cdot 0}+\theta w_{b \cdot 1}+\bar{\theta}\left(w_{b \cdot 3}-\theta w_{b \cdot 2}\right) \\
& w_{f}=w_{f}^{p}+\bar{\theta} w_{f}^{Q}=w_{f \cdot 1}+\theta w_{f \cdot 0}+\bar{\theta}\left(w_{f \cdot 2}-\theta w_{f \cdot 3}\right) .
\end{aligned}
$$

Equations (2.2) give the following equations for $w^{P}=\left(w_{b}^{P}, w_{f}^{P}\right)$ :

$$
\begin{aligned}
& \bar{\partial}_{z} w_{b \cdot 0}=\mu_{0} \partial_{z} w_{b \cdot 0}+\frac{1}{2} \kappa w_{f \cdot 1}\left(w_{f \cdot 0}+v_{1} \partial_{z} w_{f \cdot 1}\right), \\
& \bar{\partial}_{z} w_{f \cdot 1}=\mu_{0} \partial_{z} w_{f \cdot 1}+\frac{1}{2} \kappa\left(w_{f \cdot 0}+v_{1} \partial_{z} w_{f \cdot 1}\right),
\end{aligned}
$$

with

$$
\kappa=\left.\eta(\mu, v)\right|_{\theta=\bar{\theta}=0}=\frac{\mu_{1}+v_{1} \partial_{z} \mu_{0}-\partial_{z} v_{1} \mu_{0}+\bar{\partial}_{z} v_{1}}{1+v_{0}+v_{1} \partial_{z} v_{1}}
$$

and

$$
\begin{aligned}
\left(w_{f \cdot 0}+v_{1} \partial_{z} w_{f \cdot 1}\right)^{2} & =\left(1+v_{0}+v_{1} \partial_{z} v_{1}\right)\left(\partial_{z} w_{b \cdot 0}+w_{f \cdot 1} \partial_{z} w_{f \cdot 1}\right), \\
w_{b \cdot 1} & =w_{f \cdot 1} w_{f \cdot 0}-\frac{v_{1}}{1+v_{0}} w_{f \cdot 0}^{2} .
\end{aligned}
$$

Note that $w^{P}$ depends on the super Beltrami differentials only through the combinations of $\mu(\varrho, v)^{P}$ and $v^{P}$. From $(2.5), w^{Q}=\left(w_{b}^{Q}, w_{f}^{Q}\right)$ is solved in terms of $w^{P}$, $\varrho^{P}$, and $v$. Let us write Eqs. (2.5) as

$$
\bar{D} w=\left[-\varrho \partial_{z}+\frac{1}{2} G(\varrho, v) D_{v}\right] w \equiv \chi(\varrho, v: w) .
$$

Then,

$$
w^{Q}=[\chi(\varrho, v: w)]^{P}=\left[\chi\left(\varrho^{P}, v: w^{P}\right)\right]^{P} .
$$

According to [11], we expand Eqs. (3.3) with respect to the Grassmann generators and look them over at each grade. Let us write the expansion of the solution $\left(w_{b \cdot 0}, w_{f \cdot 1}\right)$ as

$$
w_{b \cdot 0}=w_{(0)}+w_{(2)}+w_{(4)}+\ldots, \quad w_{f \cdot 1}=w_{(1)}+w_{(3)}+w_{(5)}+\ldots .
$$


The body part of Eq. (3.3a) is an ordinary Beltrami equation for $w_{(0)}$. At each grade $n(\geqq 1)$, Eqs. (3.3) gives an inhomogeneous Beltrami equation for $w_{(n)}$, which involves terms depending on the solution $w_{(m)}$ of lower levels $(m<n)$. Therefore we can solve Eqs. (3.3) recursively. In [11], the boundary conditions

$$
\begin{array}{ll}
w_{(0)}(0)=0, & w_{(0)}(\infty)=\infty, \quad w_{(0)}(1)=1, \\
w_{(n)}(0)=0, & w_{(n)}(\infty)<\infty, \quad \text { for } \quad n \geqq 1,
\end{array}
$$

were considered. Under (3.6), each (inhomogeneous) Beltrami equation has a unique solution [22]. However, it should be noticed that the conditions of the soul part of $w_{b \cdot 0}$, (i.e. $\left.w_{(2 n)}, n \geqq 1\right)$ at infinity are not essential and conventional ones. The solution $w$ obtained from (3.3) (3.5) and normalized by (3.6) gives a superdiffeomorphism of $S \mathbb{C}$ such that can be extended to that of $S \mathbb{C}$, satisfying the following condition ${ }^{11}$ :

$$
w(0,0)=(0,0), \quad w(\infty, 0)=(\infty, 0), \quad \pi\left(w_{b}(1,0)\right)=1 .
$$

We see that the condition (3.7) itself is still preserved under the dilatation

$$
D_{[k]}:\left(w_{b}, w_{f}\right) \rightarrow\left(k^{2} w_{b}, k w_{f}\right)
$$

with constant $k$ of $\pi(k)=1$, and hence less restrictive than (3.6). We also notice that (3.7) is preserved under the composition of maps, that is, if $w^{i}(i=1,2)$ satisfies (3.7), so does $w^{2} \circ w^{1}$. The condition (3.7) is canonical but does not fix the solution completely. Here instead of (3.6), we adopt another condition:

$$
w(0,0)=(0,0), \quad w(\infty, 0)=(\infty, 0), \quad w_{b}(1,0)=1 .
$$

This can be achieved from (3.7) by making use of $D_{[k]}$. Once the boundary condition was set up, we have precisely two solutions each corresponding to the choice of sign of the square root appearing in (3.3c). We write one of them as $w^{\Psi}=\left(w_{b}^{\Psi}, w_{f}^{\Psi}\right)$, then the other is $I \circ w^{\Psi}=\left(w_{b}^{\Psi},-w_{f}^{\Psi}\right)$. We call them the standard solutions.

Let $\mathrm{SH}^{*}$ be the superlower half-plane: $\{(z, \theta) \mid \operatorname{Im} \pi(z)<0\}$. If $\Psi=(\varrho, v) \in \mathscr{M}(\mathrm{SH})$, the structure $\Psi$ can be extended to $\hat{\Psi}$ defined on the whole plane $S \mathbb{C}$ by the reflection:

$$
\hat{\Psi}=(\hat{\varrho}, \hat{v})=\left\{\begin{array}{lll}
(\varrho(z, \theta), v(z, \theta)) & \text { for } \quad(z, \theta) \in \mathrm{SH}, \\
(\varrho(\bar{z}, \bar{\theta}), & \overline{v(\bar{z}, \bar{\theta})}) & \text { for } \quad(z, \theta) \in \mathrm{SH}^{*} .
\end{array}\right.
$$

The reflection symmetry and the uniqueness (up to the composition with $I$ ) of the solution imply that $w^{\hat{\Psi}}$ satisfies $^{12}$

$$
w^{\hat{\Psi}}(z, \theta)=\overline{w^{\hat{\Psi}}(\bar{z}, \bar{\theta})} \text {. }
$$

Then the map $w^{\hat{\Psi}}$ defines a quasisuperconformal map of SH onto itself which can be extended to $\partial \mathrm{SH} \cup(\infty, 0)$ satisfying the boundary condition (3.8). In the following, we write simply $w^{\Psi}$ for $w^{\hat{\Psi}}$. After these studies of the quasisuperconformal maps, we can describe the deformations of SRS's as the deformations of the super Fuchsian groups.

${ }^{11}$ Here $(\infty, 0)$ is considered as a point of $S \hat{\mathbb{C}}$. Note that $(\infty, 0)$ and $(\infty, \alpha)$ with odd constant $\alpha$ are the same point in $S \hat{\mathbb{C}}$, remembering that the proper coordinate transformation around the infinity is $(z, \theta) \rightarrow(\tilde{z}, \widetilde{\theta})=\left(-\frac{1}{z}, \frac{\theta}{z}\right)$

12 If $I \circ w^{\hat{\Psi}}(z, \theta)=\overline{w^{\hat{\Psi}}(\bar{z}, \bar{\theta})}$, it leads to a contradiction when we deform $(\varrho, v)$ continuously to $(0,0)$. For $w^{\hat{\Psi}}$ turns to the identity map or the map $I$ 
If $\Psi=(\varrho, v) \in \mathrm{SB}(\Gamma)_{b}$, it is a consequence of (2.1) that $w^{\Psi}$ and $w^{\Psi} \circ A, A \in \Gamma$ satisfy the same super Beltrami equation (2.2). From (2.1) and (2.2), one can show directly that $w^{\Psi} \circ A$ is a superconformal transform of $w^{\Psi}$ :

$$
w^{\Psi} \circ A=A^{\Psi} \circ w^{\Psi}
$$

with $A^{\Psi} \in \operatorname{Aut}(\mathrm{SH})$. Moreover it could be shown that $A^{\Psi}$ is an element of $\operatorname{SPL}(2, \mathbb{R})$ as follows. The map $w^{\Psi}$ gives a superdiffeomorphism of $S \hat{\mathbb{C}}$. Thus $A^{\Psi}=w^{\Psi} \circ A \circ w^{i F^{-1}}$ is an element of $\operatorname{SPL}(2, \mathbb{C})$. The reflection symmetry (3.10) implies that $A^{\Psi}$ fixes $S \mathbb{R}$ :

$$
A^{\Psi}(z, \theta)=\overline{A^{\Psi}(\bar{z}, \bar{\theta})}
$$

from which we conclude $A^{\Psi} \in \operatorname{SPL}(2, \mathbb{R})$. However, there exist subtle problems. For the reasoning made above to be correct, (3.7) must be well defined at $S \mathbb{R}$. Discontinuities of $\Psi$ lying across $\partial \mathrm{SH}$, which may occur by (3.9) in general, descend to the solution $w^{\Psi}$ (except its first components $\left(w_{b \cdot 0}, w_{f \cdot 1}\right)$, which will be continuous due to the good properties of the ordinary (inhomogeneous) Beltrami equation [22]). For a non-split $\Gamma$, the discontinuities affect $A^{\Psi}$ directly. We discuss these points in Appendix A. If one takes a split $\Gamma$ as the initial point, however, there arise no problems since then $A^{\Psi}$ is determined only through the first components of $w^{\Psi}$, as we will see in the next section.

An isomorphism $\vartheta^{\Psi}$ from $\Gamma_{p}$ into $\operatorname{SPL}(2, \mathbb{R})$ is obtained by

$$
\vartheta^{\Psi}(a)=w^{\Psi} \circ \vartheta(a) \circ w^{\Psi-1}, \quad a \in \Gamma_{p},
$$

where $\vartheta$ is the normalized isomorphism which maps $\Gamma_{p}$ on $\Gamma$. Evidently $\Gamma^{\Psi}=\vartheta^{\Psi}\left(\Gamma_{p}\right)$ is a super Fuchsian group which represents the deformed SRS $\Sigma^{\Psi}=\mathrm{SH} / \Gamma^{\Psi}$. Here some comments on the normalization of $\vartheta^{\Psi}$ should be made. Since $w^{\Psi}$ satisfies (3.7), $\vartheta^{\Psi}$ is almost normalized, but not completely, i.e. $\vartheta^{\Psi}\left(a_{1}\right)$ has fixed point at $(0,0)$, $(\infty, 0)$, and $\pi\left(\vartheta_{b}^{\Psi}\left(a_{2}\right)(1,0)\right)=1$ is satisfied but not $\vartheta_{b}^{\Psi}\left(a_{2}\right)(1,0)=1$ in general. There is no canonical normalization for the quasisuperconformal maps to keep $w \circ \Gamma \circ w^{-1}$ completely normalized. Our choice for $w^{\Psi}$ is the condition (3.8). To make $\vartheta^{\Psi}$ completely normalized, we must shift $w^{\Psi}$ to $D_{[k]} \circ w^{\Psi}$ in (3.12), choosing the dilatation constant $k(\pi(k)=1)$ properly for each $\Psi$.

The following statement is a super-analogue concerning the Teichmüller equivalence relation and can be proved in the almost same way.

Proposition 3.1. Let $\Psi, \Psi^{\prime} \in \operatorname{SB}(\Gamma)_{b}$. Then $\Psi \sim \Psi^{\prime}$ if and only if $\vartheta^{\Psi}=\vartheta^{\Psi^{\prime}}$, modulo conjugations by $D_{[k]}$ with $\pi(k)= \pm 1$.

Note that $D_{[-1]}=I$. Due to the existence of the two solutions under (3.8), $\vartheta^{\Psi}$ is primarily defined up to the conjugation by $I$. The resulting ambiguity peculiar to the super Fuchsian group has been already mentioned. What about if we represent the initial SRS by SH/III? The answer is the following.

Proposition 3.2. Let $\Psi=(\varrho, v) \in \mathrm{SB}(\Gamma)_{b}$. Then $-\Psi \circ I=(-\varrho \circ I,-v \circ I)$ is an element of $\mathrm{SB}(I \Gamma I)_{b}$ and $\mu(-\varrho \circ I,-v \circ I)=\mu(\varrho, v) \circ I$. The standard solution is given by $w^{-\Psi \circ I}=I \circ w^{\Psi} \circ I$.

Proof. For any superfunction, we have $D_{-v \circ I}(F \circ I)=-\left(D_{v} F\right) \circ I$, where the minus sign of $-v \circ I$ is necessary. In particular,

$$
D_{-v \circ I}\left((I \circ A \circ I)_{f}\right)=\left(D_{v} A_{f}\right) \circ I .
$$

Taking account of these formulas, we obtain the desired result. Q.E.D. 
Obviously, $\Psi$ and $-\Psi \circ I$ describe the same deformation and give the correspondence between the $\operatorname{SB}(\Gamma)_{b}$ and $\mathrm{SB}(I \Gamma I)_{b}$. We call a super Fuchsian group $\Gamma$ split if $I \Gamma I=\Gamma$, that is, all the odd parameters of the generators of $\Gamma$ vanish. Then $\mathrm{SH} / \Gamma$ becomes a split SRS. If we take a split SRS as the initial point, we obtain an interesting result as a corollary.

Corollary 3.3. Let $\Gamma$ be a split Super Fuchsian group. If $\Psi \in \operatorname{SB}(\Gamma)_{b}$, then $-\Psi \circ I \in \mathrm{SB}(\Gamma)_{b}$ and $-\Psi \circ I \sim \Psi$.

The transformation $\Psi \rightarrow-\Psi \circ I$ (then $\mu \rightarrow \mu \circ I$ ) changes the signs of the odd components of the super Beltrami differentials. The symmetry under this transformation enters into the super Teichmüller space $\mathrm{SB}(\Gamma)_{b} / \sim$ and brings the super-orbifold structure.

\section{Special Gauge Choice}

In this section, we study the special choice of gauge for the super Beltrami differentials conjectured in $[11,12]$ and prove that this gauge choice is always possible. It simplifies the various expressions in the previous sections. To start with, we want to investigate the formula (3.11). Using the previous notations, we separate the solution $w^{\Psi}$ into two parts:

$$
w^{P}=\left(w_{b}^{P}, w_{f}^{P}\right), \quad w^{Q}=\left(w_{b}^{Q}, w_{f}^{Q}\right),
$$

and occasionally treat them like vector functions. Remember that $w^{Q}$ has the expression given by (3.4). The superscript $\Psi$ of $w^{\Psi}$ is often omitted for simplicity. An element $A$ of the super Fuchsian group is expanded in terms of $\theta$ as

$$
A(z, \theta)=\left(A_{b \cdot 0}(z)+\theta A_{b \cdot 1}(z), A_{f \cdot 1}(z)+\theta A_{f \cdot 0}(z)\right) .
$$

Proposition 4.1. Let $\Psi=(\varrho, v) \in \mathrm{SB}(\Gamma)_{b} . A^{\Psi}$ is determined only by the $\bar{\theta}$-free part of

$$
A^{\Psi} \circ w=w \circ A \text {, }
$$

that is

$$
A^{\Psi} \circ w^{P}=w^{P} \circ A+\overline{A_{f \cdot 1}}\{(\bar{D} w) \circ A\} .
$$

Moreover, if $\Gamma$ is a split super Fuchsian group, i.e. $A_{f \cdot 1}=A_{b \cdot 1}=0$ for every $A \in \Gamma$, then $A^{\Psi}$ is determined except the choice of sign of $A_{f \cdot 0}^{\Psi}$ only by the first components of $w^{\Psi}$, i.e. $\left(w_{b \cdot 0}, w_{f \cdot 1}\right)$.

Proof. First, we show that the identity obtained from the $\bar{\theta}$ coefficients of (4.1) is simply a consequence of (4.2). Thus (4.1) has no additional information more than (4.2). The $\bar{\theta}$-containing part of the left-hand side of (4.1) is

$$
\bar{\theta} w_{b}^{Q}\left\{\left(A_{0}^{\Psi}\right)^{\prime} \circ w_{b}^{P}+w_{f}^{P}\left\{\left(A_{1}^{\Psi}\right)^{\prime} \circ w_{b}^{P}\right\}\right\}+\bar{\theta} w_{f}^{Q}\left(A_{1}^{\Psi} \circ w_{b}^{P}\right),
$$

where $A_{0}(z)=\left(A_{b \cdot 0}(z), A_{f \cdot 1}(z)\right)$ and $A_{1}(z)=\left(A_{b \cdot 1}(z), A_{f \cdot 0}(z)\right)$.

Using (2.2a), $w_{b}^{Q}$ is expressed by $w_{f}^{Q}$ as

$$
w_{b}^{Q}=\left[\chi\left(\varrho, v: w_{b}\right)\right]^{P}=w_{f}^{P}\left[\chi\left(\varrho, v: w_{f}\right)\right]^{P}-\left[\varrho \frac{\left(D_{v} w_{f}\right)^{2}}{D_{v} \hat{v}}\right]^{P},
$$

thus the expression becomes

$$
\bar{\theta} \chi\left(\varrho, v: w_{f}\right)\left\{\left(D A^{\Psi}\right) \circ w^{P}\right\}-\bar{\theta} \varrho \frac{\left(D_{v} w_{f}\right)^{2}}{D_{v} \hat{v}}\left\{\left(\partial_{z} A^{\Psi}\right) \circ w^{P}\right\}
$$


The $\bar{\theta}$-containing part of the right-hand side of (4.1) has the following form:

$$
\bar{\theta} \chi(\varrho, v: w \circ A)=\bar{\theta} \chi\left(\varrho, v: w^{P} \circ A\right)-\overline{\theta A_{f \cdot 1}} \chi\left(\varrho, v: w^{Q} \circ A\right) .
$$

Here we use the identity $[w \circ A]^{Q}=[\chi(\varrho, v: w \circ A)]^{P}$, which comes from the fact that $w \circ A$ satisfies the same super Beltrami equation. By differentiating (4.2), we have

$$
\begin{aligned}
\bar{\theta} D_{v}\left(A^{\Psi} \circ w^{P}\right) & =\bar{\theta} D_{v}\left(w^{P} \circ A\right)-\bar{\theta} \overline{A_{f \cdot 1}} D_{v}\left(w^{Q} \circ A\right), \\
\bar{\theta} \partial_{z}\left(A^{\Psi} \circ w^{P}\right) & =\bar{\theta} \partial_{z}\left(w^{P} \circ A\right)+\bar{\theta} \overline{A_{f \cdot 1}} \partial_{z}\left(w^{Q} \circ A\right) .
\end{aligned}
$$

We should also notice the following identities:

$$
\begin{gathered}
\left(D A^{\Psi}\right) \circ w=\frac{1}{D_{v} w_{f}} D_{v}\left(A^{\Psi} \circ w\right), \\
\frac{\left(D_{v} w_{f}\right)^{2}}{D_{v} \hat{v}}\left\{\left(\partial_{z} A^{\Psi}\right) \circ w\right\}=\partial_{z}\left(A^{\Psi} \circ w\right)-\partial_{z} w_{f}\left\{\left(D A^{\Psi}\right) \circ w\right\} .
\end{gathered}
$$

(Under the multiplication by $\bar{\theta}$, we can replace $w$ with $w^{P}$.) Substituting these expressions into (4.3) and taking account of the explicit form of $\chi(\varrho, v:)$, we obtain the equality between (4.3) and (4.4). Next, suppose that $\Gamma$ is split. Then the $\theta$-free part of (4.2) becomes as follows:

$$
\begin{aligned}
& A_{b \cdot 0}^{\Psi} \circ w_{b \cdot 0}+w_{f \cdot 1}\left(A_{b \cdot 1}^{\Psi} \circ w_{b \cdot 0}\right)=w_{b \cdot 0} \circ A_{b \cdot 0}, \\
& A_{f \cdot 1}^{\Psi} \circ w_{b \cdot 0}+w_{f \cdot 1}\left(A_{f \cdot 0}^{\Psi} \circ w_{b \cdot 0}\right)=w_{f \cdot 1} \circ A_{b \cdot 0} .
\end{aligned}
$$

Remember that $A_{b \cdot 1}^{\Psi}=A_{f \cdot 1}^{\Psi} A_{f \cdot 0}^{\Psi}$ and $A_{f \cdot 0}^{\Psi}{ }^{2}=\left(A_{b \cdot 0}^{\Psi}\right)^{\prime}+A_{f \cdot 1}^{\Psi}\left(A_{f \cdot 1}^{\Psi}\right)^{\prime}$. Once the choice of the sign of $A_{f \cdot 0}^{\Psi}$ is fixed, (4.5) determines $A^{\Psi}$ order by order with respect to the Grassmann generators. Starting to find the body part of $A_{b \cdot 0}^{\Psi}$, we can obtain the successive higher order terms of $A^{\Psi}$ inductively. Q.E.D.

The spin structure associated with $\mathrm{SH} / \Gamma$ is specified by the signs (positive or negative) of $\pi\left(A_{f \cdot 0}^{(i)}\right)$ at the fixed point of $A_{b \cdot 0}^{(i)}$, where $A^{(i)}=\vartheta\left(a_{i}\right)$ are the generators of $\Gamma .^{13}$ If we replace the condition $\left(2.9 \mathrm{a}^{\prime}\right)$ with more weakly one (2.9a), we have the following proposition.

Proposition 4.2. The spin structures of $\Sigma^{\Psi}$ are classified by the homotopy class of $\pi\left(1+v_{0}\right)$ modulo $(2 \mathbb{Z})^{2 p}$ (as a map from $\pi(\Sigma)$ to $\mathbb{C}-\{0\}$ ).

Proof. The sign of $A_{f \cdot 0}^{\Psi}$ at the fixed points is determined through the $\theta$-containing part of (4.2). Neglecting the nilpotent terms, we have

$$
\pi\left(w_{f \cdot 0}\left(A_{f \cdot 0}^{\Psi} \circ w_{b \cdot 0}\right)\right)=\pi\left(A_{f \cdot 0}\left(w_{f \cdot 0} \circ A_{b \cdot 0}\right)\right) .
$$

From (3.3c), it becomes

$$
\pi\left(A_{f \cdot 0}^{\Psi} \circ w_{b \cdot 0}\right)=\pi\left(\frac{\left(1+v_{0}\right)^{1 / 2} \circ A_{b \cdot 0}}{\left(1+v_{0}\right)^{1 / 2}}\right) \pi\left(A_{f \cdot 0} \frac{\left(\partial_{z} w_{b \cdot 0}\right)^{1 / 2} \circ A_{b \cdot 0}}{\left(\partial_{z} w_{b \cdot 0}\right)^{1 / 2}}\right) .
$$

${ }^{13}$ For an element $A$ of $\Gamma, A_{b, 0}(z)$ is written as

$$
A_{b} \cdot 0(z)=\frac{a z+b}{c z+d}, \quad a d-b c=1, \quad|\pi(a+b)|>2 .
$$

Denote its fixed points by $u_{ \pm}$. Then

$$
\pi\left(A_{f} \cdot 0\left(u_{ \pm}\right)\right)=\pi\left(\frac{1}{c u_{ \pm}+d}\right)=\pi\left(\frac{1}{2}\left\{(a+d) \pm \sqrt{(a+d)^{2}-4}\right\}\right)\left\{\begin{array}{lll}
>0 & \text { for } & \pi(a+b)>2 \\
<0 & \text { for } & \pi(a+b)<-2
\end{array} .\right.
$$


Note that $\pi\left(\left(1+v_{0}\right) \circ A_{b} \cdot 0\right)=\pi\left(1+v_{0}\right)$ from (2.1). Thus the factor appeared in the right-hand side equals to 1 or -1 and counts the twist $\bmod 2$ around the cycle represented by the element $A(\in \Gamma)$. Since $w_{b .0}$ is homotopic to the identity map on $H$, the sign of $A_{f \cdot 0}^{\Psi}$ at the fixed point (which is homotopy invariant) differs from that of $A_{f \cdot 0}$ by that factor. Q.E.D.

Now we can get the desired result about the gauge choice, restricting ourselves to the case that the initial SRS is split.

Theorem 4.3. Let $\Gamma$ be a split super Fuchsian group. Then for any deformation of $\mathrm{SH} / \Gamma$, the special gauge choice such that $\left(1-\overline{\theta \partial_{\theta}}\right) \varrho=0$ and $\nu=0$ is possible. Especially, for any $\Psi=(\varrho, v) \in \mathrm{SB}(\Gamma)_{b}$, there exists a super Beltrami differential $(-\bar{\theta} \tilde{\mu}, 0) \in \mathrm{SB}(\Gamma)_{b}$ such that $w^{(-\bar{\theta} \tilde{\mu}, 0)}$ has the first components equal to those of $w^{\Psi}$ and hence $\Psi \sim(-\bar{\theta} \tilde{\mu}, 0)$. Such $\tilde{\mu}$ is given by

$$
\begin{gathered}
\tilde{\mu}(\varrho, v)=\mu_{0}+\theta \tilde{\mu}_{1}, \\
\left.\mu_{0} \equiv \mu(\varrho, v)\right|_{\theta=\bar{\theta}=0}, \quad \tilde{\mu}_{1} \equiv\left(D_{v} \hat{v}\right)^{1 / 2} \eta(\mu(\varrho, v), v)_{\theta=\bar{\theta}=0} .
\end{gathered}
$$

Proof. It is not necessarily a trivial thing that $(-\bar{\theta} \tilde{\mu}, 0) \in \mathrm{SB}(\Gamma)_{b}$. By examining (2.1) for a split $\Gamma$, we see that $\tilde{\mu}$ becomes indeed a $(-1,1)$ superconformal tensor. Equations (3.3) shows that the first components of $w^{\Psi}$ depends upon only $\tilde{\mu}$. Since $\tilde{\mu}(-\tilde{\theta} \tilde{\mu}, 0)=\tilde{\mu}$, from Proposition 4.1 and 4.2 , we conclude that $(-\bar{\theta} \tilde{\mu}, 0)$ define the same $\vartheta^{\Psi}$. We also notice that the choice of the sign of $\tilde{\mu}_{1}$ is irrelevant because of Corollary 3.3. Q.E.D.

We refer to this special gauge as Wess-Zumino gauge according to the usage.

As a intermediate stage of this special gauge choice, we see that for any $\Psi=(\varrho, v) \in \mathrm{SB}(\Gamma)_{b}$,

$$
\Psi \sim\left(-\bar{\theta} \mu(\varrho, v), v^{P}\right)
$$

because of the identity $\mu\left(-\bar{\theta} \mu(\varrho, v), v^{P}\right)=\mu(\varrho, v)^{P}$. This is the $\sigma=0$ gauge conjectured in [11]. In regard to the $\bar{\theta}$-free part, $\left(-\bar{\theta} \mu(\varrho, v), v^{P}\right)$ and $\Psi$ give the same solution $\left(w^{\Psi}\right)^{P}$, though $\left(-\bar{\theta} \mu(\varrho, v), v^{P}\right)$ gives

$$
\bar{\theta} w^{Q}=\bar{\theta} \chi\left(-\bar{\theta} \mu, v^{P}:\left(w^{\Psi}\right)^{P}\right)=0 .
$$

What can we tell about the case that the initial $\Gamma$ is non-split? Studies of the infinitesimal deformations $[12,13]$ imply that the $v=0$ gauge is always possible. However, some plausible assumptions are needed there as to the boundary behaviors of the super Beltrami differentials. We will state these points in Appendix A. As to the $\sigma(\varrho, v)(=\varrho+\bar{\theta} \mu(\varrho, v))=0$ gauge, I'm not aware whether the corresponding gauge condition for general $\Gamma$ 's can be written in a simple form. ${ }^{14}$

\section{Appendix A. Infinitesimal Deformations and the $v=0$ Gauge}

The infinitesimal deformations of SRS have been studied by several authors $[12,13]$, however, the subject seems not completed. In this appendix, we give a description, suitably extending the arguments for non-super theory $[20,24]$, and attend to the possibility of the $v=0$ gauge for the deformations of a general (nonsplit) SRS.

${ }^{14}$ As such a candidate, one may consider the condition $\mu(\varrho, v)+\bar{D} \varrho=0 . \mu+\bar{D} \varrho$ is a $(-1,1)$ superconformal tensor, and hence this condition is covariant 
Consider a function $F(\Psi)$ defined on $\operatorname{SB}(\Gamma)_{b}$. The differentiation of $F(\Psi)$ in a direction $\Phi \in \mathrm{SB}(\Gamma)$ is defined through the expansion with respect to an infinitesimal parameter $\varepsilon$ :

$$
F(\Psi+\varepsilon \Phi)=F(\Psi)+\varepsilon \dot{F}(\Psi)[\Phi]+O\left(\varepsilon^{2}\right) .
$$

The derivative at $\Psi=0$, i.e. $\dot{F}(0)[\Phi]$ is denoted simply by $\dot{F}[\Phi]$. Note that $\varepsilon \Phi$ should be Grassmann odd, however, $\Phi$ (and hence $\varepsilon$ ) may take both odd and even parities. It is convenient to introduce a function $F_{\Psi}$ defined on $\operatorname{SB}\left(\Gamma^{\Psi}\right)_{b}$ by the identity $F_{\Psi}(\Lambda)=F(\Lambda * \Psi)$. Then $\dot{F}(\Psi)[\Phi]$ can be converted to the derivative of $F_{\Psi}$ at $\Lambda=0$ :

$$
\dot{F}(\Psi)[\Phi]=\dot{F}_{\Psi}\left[L_{\Psi}(\Phi)\right],
$$

where $L_{\Psi}(\Phi)$ is defined through

$$
\Psi+\varepsilon \Phi=\left\{\varepsilon L_{\Psi}(\Phi)+O\left(\varepsilon^{2}\right)\right\} * \Psi .
$$

Inverting the formula (2.10), we can obtain the expression of $L_{\Psi}(\Phi)$ from

$$
\left(\dot{\mu}\left[L_{\Psi}(\Phi)\right], L_{\Psi}(\Phi)\right) \circ w^{\Psi}=(\dot{\mu}(\Psi)[\Phi], \Phi)(F(\Psi))^{-1},
$$

where $\dot{\mu}$ is defined through (2.3), and

$$
F(\Psi)=\left[\begin{array}{ccc}
(1-\mu \bar{\mu}) \bar{H} & (\bar{v}-\varrho \bar{\mu}) \bar{H} & (\bar{\varrho}-v \bar{\mu}) \bar{H} \\
\left(\bar{\partial}_{z}-\mu \partial_{z}\right) \overline{w_{f}} & \left(\bar{D}+\varrho \partial_{z}\right) \overline{w_{f}} & D_{v} \overline{w_{f}} \\
\left(\bar{\partial}_{z}-\mu \partial_{z}\right) w_{f} & \left(\bar{D}+\varrho \partial_{z}\right) w_{f} & D_{v} w_{f}
\end{array}\right],
$$

with $\Psi=(\varrho, v)$ and $w^{\Psi}=\left(w_{b}, w_{f}\right)$. Apparently, the operator $L_{\Psi}$ is linear with respect to $\Phi$ in the complex sense. From these remarks, derivatives at arbitrary points can be reduced to the derivatives at origin by shifting the initial origin and replacing $\Phi$ to $L_{\psi}(\Phi)$. So, in the following, we limit our consideration to the initial derivatives $\dot{F}[\Phi]$.

Now, consider the derivative at origin of the quasisuperconformal map

$$
\dot{w}[\Phi]=\left(\dot{w}_{b}[\Phi], \dot{w}_{f}[\Phi]\right) \quad \text { with } \quad \varepsilon \Phi=(\varepsilon \check{\varrho}, \varepsilon \check{v})
$$

being an infinitesimal element of $\operatorname{SB}(\Gamma)$. We define a superfunction

$$
W[\Phi]=\dot{w}_{b}[\Phi]-\dot{w}_{f}[\Phi] \theta,
$$

which simplifies the description. Because of $(2.2 \mathrm{~b}), \dot{w}[\Phi]$ is expressed in terms of $W$ :

$$
\begin{aligned}
& \dot{w}_{f}[\Phi]=\frac{1}{2}\left\{\check{v}-(-1)^{|\Phi|} D W[\Phi]\right\}, \\
& \dot{w}_{b}[\Phi]=W[\Phi]+\dot{w}_{f}[\Phi] \theta .
\end{aligned}
$$

$W[\Phi]$ satisfies the linearized super Beltrami equation which is given by

$$
\bar{D} W[\Phi]=(-1)^{|\Phi|} \varrho .
$$

The boundary condition for $W[\Phi]$ can be found by noting

$$
\begin{aligned}
W(z, 0) & =\dot{w}_{b \cdot 0}(z), \\
D W(z, 0) & =\dot{w}_{b \cdot 1}(z)+\dot{w}_{f \cdot 1}(z)=2 \dot{w}_{f \cdot 1}-\check{v},
\end{aligned}
$$


and from (3.7) [13],

$$
\begin{aligned}
& w_{b \cdot 0}(0)=w_{b \cdot 0}(1)=0 \text {, } \\
& w_{f \cdot 1}(0)=0, \\
& \left.\begin{array}{l}
\frac{w_{b \cdot 0}(z)}{z^{2}} \rightarrow 0 \\
\frac{w_{f \cdot 1}(z)}{z} \rightarrow 0
\end{array}\right\} \text { for } z \rightarrow \infty \text {. }
\end{aligned}
$$

Before trying to find the expression of the solution, we consider the corresponding (infinitesimal) deformation of the super Fuchsian group:

$$
A \rightarrow A+\varepsilon \dot{A}[\Phi], \quad A \in \Gamma \text {. }
$$

From the definition of $A^{\Psi}$, we obtain ${ }^{15}$

$$
\dot{w}[\Phi] \circ A=\dot{A}[\Phi]+W[\Phi] \partial_{z} A+\dot{w}_{f}[\Phi] D A .
$$

From $(\mathrm{A} .3 \sim 5)$ and the transformation properties of $\Phi$ under $\Gamma$, we see that

$$
\begin{gathered}
\bar{D} \dot{A}[\Phi]=0, \\
(-1)^{|\Phi|} D \dot{A}_{b}[\Phi]+\dot{A}_{f}[\Phi] D A_{f}+A_{f} D \dot{A}_{f}[\Phi]=0,
\end{gathered}
$$

which show that the superconformality of $A$ is preserved under the deformation. Instead of $\dot{A}[\Phi]$, it is convenient to introduce a function $V_{A}[\Phi]$ just like $W[\Phi]$ :

$$
V_{A}[\Phi]=\dot{A}_{b}[\Phi]-\dot{A}_{f}[\Phi] A_{f} .
$$

Then $\bar{D} V_{A}[\Phi]=0$ and $\dot{A}[\Phi]$ is expressed by $V_{A}[\Phi]$ because of (A.10):

$$
\begin{aligned}
& \dot{A}_{f}[\Phi]=-(-1)^{|\Phi|} \frac{1}{2} \frac{D V_{A}[\Phi]}{D A_{f}}, \\
& \dot{A}_{b}[\Phi]=V_{A}[\Phi]+\dot{A}_{f}[\Phi] A_{f} .
\end{aligned}
$$

We can rewrite (A.8) as follows:

$$
\begin{aligned}
V_{A}[\Phi] & =W[\Phi] \circ A-\left(D A_{f}\right)^{2} W[\Phi] \\
& =W[\Phi]^{P} \circ A-\left(D A_{f}\right)^{2} W[\Phi]^{p}+\check{\varrho} \frac{\left(D A_{f}\right)^{2}}{D A_{f}} A_{f, 1},
\end{aligned}
$$

where $W^{P}$ denotes the $\bar{\theta}$-free part of $W$.

Equation (4.5) and the formulas (A.12,13) imply that if $\dot{A}[\Phi]$ depends on $\check{v}$, it does only through the boundary condition. Moreover (A.6) and (A.13) show that we encounter with the difficulties mentioned in Sect. 3, that is, the discontinuities of $\Phi$ lying across $\partial \mathrm{SH}$ affect $\dot{A}[\Phi]$ directly. As a result, the deformation of $\Gamma$ as a subgroup of $\operatorname{SPL}(2, \mathbb{R})$ becomes dubious. For a split $\Gamma$, this is not the case. Being required the smoothness on $\mathrm{SH}$ (and hence on $\mathrm{SH}^{*}$ ), $\Phi$ should be extended to $\partial \mathrm{SH}$, then the odd components of $\Phi$ have to vanish on $\mathbb{R}$ because of the transformation laws (2.1). For example, in this case, $\check{v}_{1}$ transforms as

$$
\check{v}_{1} \circ A_{b \cdot 0}=\left(A_{b \cdot 0}^{\prime}\right)^{1 / 2} \check{v}_{1}, \text { for } A \in \Gamma \text {. }
$$

\footnotetext{
${ }^{15}$ Here we use the notation in which $w=\left(w_{b}, w_{f}\right)$ and $A=\left(A_{b}, A_{f}\right)$ and their derivatives are dealt like vector functions
} 
This shows " $\check{v}_{1}$ vanishes at the fixed points $u_{ \pm}$of $A_{b \cdot 0}$, because $A_{b \cdot 0}^{\prime}\left(u_{ \pm}\right) \neq 1$ [provided that $A_{b \cdot 0}$ is a hyperbolic element of $\left.\mathrm{SL}(2, \mathbb{R})\right]$. Since these fixed points are dense on $\mathbb{R}, \check{v}_{1}$ must vanish on $\mathbb{R}$. The same holds true for the other odd components of $\Phi$. For the case of non-split $\Gamma$, it appears that vanishing on $\mathbb{R}$ of the first odd components $\left(\check{\varrho}_{1}, \check{v}_{1}\right)$ implies

$$
\left(\check{\varrho}_{0}+\check{\varrho}_{2}, \check{v}_{0}+\check{v}_{2}\right)=0 \text { on } \mathbb{R}
$$

because the condition of vanishing on $S \mathbb{R}$ is covariant. It is not obvious whether the same reasoning mentioned above leads the result $\Phi=0$ on $S \mathbb{R} .{ }^{16}$ Anyway we may have to make the following assumption [13].

Assumption A.1. The space of super Beltrami differentials $\mathrm{SB}(\Gamma)_{b}$ consists of the elements such that are smooth and become zero on $S \mathbb{R}$.

It is probable that to cover the whole deformations of SRS, it is sufficient to consider such a class of super Beltrami differentials. Admitting this assumption, $W[\Phi]$ does not depend on $\check{v}$, in addition, $W^{P}[\Phi]$ has only dependence on $\bar{\partial}_{\theta} \varrho$ and so does $V_{A}[\Phi]$ from (A.12) since $V_{A}[\Phi]$ is determined by its values on $S \mathbb{R}$. It becomes an immediate result that the $v=0$ gauge is always possible. Let $\Psi=(\varrho, v)$ be an element of $\mathrm{SB}(\Gamma)_{b}$. From (A.5) and (A.13), $(0, \lambda) \in \mathrm{SB}\left(\Gamma^{\Psi}\right)$ gives a stationary direction at $\Gamma^{\Psi}$, i.e. $\left(\dot{A}^{\Psi}\right)[(0, \lambda)]=0$ for all $A^{\Psi} \in \Gamma^{\Psi}$. Let $\Phi=(\check{\varrho}, \grave{v}) \in \mathrm{SB}(\Gamma)$ such that $(0, \lambda)=L_{\Psi}(\Phi)$. Then from (A.2), we have

$$
\begin{aligned}
& \check{\varrho}=\frac{1}{2}\left(\lambda \circ w^{\Psi}\right) G(\Psi) \frac{D_{v} \hat{v}}{D_{v} w_{f}^{\Psi}}, \\
& \check{v}=\left(\lambda \circ w^{\Psi}\right) \frac{D_{v} \hat{v}}{D_{v} w_{f}^{\Psi}},
\end{aligned}
$$

and hence

$$
\check{\varrho}=\frac{1}{2} \check{\nu} G(\Psi) \text {. }
$$

In addition, it follows that

$$
\dot{\mu}(\Psi)[\Phi]=\frac{1}{2} \check{\nu} \eta(\Psi) .
$$

Since one can take the differential $\check{v}$ arbitrary by varying $\lambda$, an infinitesimal variation of

$$
\Psi: \Psi \rightarrow \Psi+\varepsilon \Phi
$$

such that $\Phi$ takes the form

$$
\Phi=\check{\nu}\left(\frac{1}{2} G(\Psi), 1\right)
$$

is an infinitesimal gauge transformation. There is no obstruction to integrate to get $v=0$, if the condition $\left(2.9 a^{\prime}\right)$ is satisfied.

${ }^{16}$ Examining the transformation law (2.1b) on $S \mathbb{R}$ (instead of $\partial \mathrm{SH}$ for simplicity), we are led to consider the following identities on $\mathbb{R}$ :

$$
\begin{gathered}
v_{1} \circ A_{b \cdot 0}=\frac{A_{b .0}^{\prime}}{A_{f \cdot 0}} v_{1}-A_{f \cdot 1}\left(v_{0}+v_{2}\right), \\
\left(v_{0}+v_{2}\right) \circ A_{b \cdot 0}=\frac{A_{f \cdot 0}^{2}}{A_{b \cdot 0}^{\prime}}\left(v_{0}+v_{2}\right)+\left(\frac{A_{f \cdot 1}}{A_{f \cdot 0}}\right)^{\prime} v_{1}-\frac{A_{f \cdot 1}}{A_{f \cdot 0}}\left[\partial_{z}+\bar{\partial}_{z}\right] v_{1}
\end{gathered}
$$


On the assumption mentioned above, the boundary conditions for $W[\Phi]$ are

$$
\begin{aligned}
& W(0,0)=W(1,0)=D W(0,0)=0, \\
& \left.\begin{array}{l}
\frac{W(z, 0)}{z^{2}} \rightarrow 0 \\
\frac{D W(z, 0)}{z} \rightarrow 0
\end{array}\right\} \text { for } z \rightarrow \infty .
\end{aligned}
$$

The solution of Eq. (4.5) with these conditions is found to be

$$
\begin{aligned}
W[\Phi](z, \theta)= & \frac{-1}{\pi} \int_{\mathrm{SH}} d^{2} u d \xi d \bar{\xi}\{\varrho(u, \xi)\{\xi R(u, z)-\theta P(u, z)\} \\
& -\bar{\varrho}(u, \xi)\{\bar{\xi} R(\bar{u}, z)-\theta P(\bar{u}, z)\}\}+\check{\varrho} \bar{\theta},
\end{aligned}
$$

where

$$
\begin{aligned}
& R(u, z)=\frac{1}{u-z}-\frac{z}{u-1}+\frac{z-1}{u}, \\
& P(u, z)=\frac{1}{u-z}-\frac{1}{u}
\end{aligned}
$$

The function $W[\Phi]$ is a linear functional of the super Beltrami differentials $\Phi$ in the real sense. We can obtain a complex antilinear functional by

$$
\begin{aligned}
& X[\Phi](z, \theta)=W[\Phi]+i W[i \Phi] \\
& =\frac{2}{\pi} \int_{\mathrm{SH}} d^{2} u d \xi d \bar{\xi} \overline{\varrho(u, \xi)}\{\bar{\xi} R(\bar{u}, z)-\theta P(\bar{u}, z)\},
\end{aligned}
$$

which becomes super-analytic: $\bar{D} X[\Phi]=0$. Let us introduce a functional

$$
\begin{aligned}
\chi[\Phi](z, \theta) & \equiv \partial_{z}^{2} D X[\Phi](z, \theta) \\
& =-(-1)^{|\Phi|} \frac{4}{\pi} \int_{\mathrm{SH}} d^{2} u d \xi d \bar{\xi} \frac{\bar{\varrho}(u, \xi)}{(\bar{u}-z-\bar{\xi} \theta)^{3}},
\end{aligned}
$$

and consider the mapping $J: \Phi \mapsto \chi[\Phi]$. By examining the transformation with respect to $\Gamma$, it is easily seen that $\chi[\Phi]$ is a superholomorphic $\left(\frac{3}{2}, 0\right)$ superconformal tensor. Let us denote by $\operatorname{SQ}(\Gamma)$ the linear space of the superholomorphic $\left(\frac{3}{2}, 0\right)$ superconformal tensors and by $\mathrm{N}(\Gamma)$ the linear space consisting of all stationary directions at origin $\mathrm{SH} / \Gamma$. The following proposition gives characterizations of $\mathrm{N}(\Gamma)$.

Proposition A.2. The following conditions are all equivalent.

(a) $V_{A}[\Phi]=0$ (i.e. $\dot{A}[\Phi]=0$ ) for all $A \in \Gamma$,

(b) $W[\Phi]=0$ on $S \mathbb{R}$,

(c) $X[\Phi]=0$ in $S H$,

(d) $\chi[\Phi]=0$ in $S H$.

On the previous assumption, the proof is a straightforward extension of the correspondent in the non-super theory [20] (see also [16]). Proposition A.2(d) shows $\mathrm{N}(\Gamma)=\operatorname{ker} J$. Thus the mapping $J$ induces an antilinear isomorphism from 
$\mathrm{SB}(\Gamma) / \mathrm{N}(\Gamma)$ into $\mathrm{SQ}(\Gamma)$. It can be also checked that for an element $S(z, \theta)$ in $\mathrm{SQ}(\Gamma)$, a mapping $J^{*}$

$$
J^{*}: S(z, \theta) \mapsto-(-1)^{|S|}\left(\frac{z-\bar{z}-\theta \bar{\theta}}{2 i}\right)^{2} \overline{S(z, \theta)}
$$

defines an antilinear mapping from $\mathrm{SQ}(\Gamma)$ to $\mathrm{SB}(\Gamma)$. We note a super-analogue of a Bergman type reproducing formula:

$$
\begin{aligned}
S(z, \theta) & =\frac{4}{\pi} \int_{\mathrm{SH}} d^{2} u d \xi d \bar{\xi} \frac{\left(\frac{z-\bar{z}-\theta \bar{\theta}}{2 i}\right)^{2} \overline{S(u, \xi)}}{(\bar{u}-z-\bar{\xi} \theta)^{3}} \\
& =\chi\left[J^{*} S\right](z, \theta),
\end{aligned}
$$

which shows $J J^{*}$ is the identity map. Therefore $J$ is also surjective. We conclude that $\mathrm{SB}(\Gamma) / \mathrm{N}(\Gamma)$, which is identified with the tangent space at origin of the super Teichmüller space $\mathrm{ST}(\Gamma)$, is isomorphic to $\mathrm{SQ}(\Gamma)$. Here we set $\mathrm{SB}(\Gamma)$ and $\mathrm{SQ}(\Gamma)$ to be those satisfying

$$
{ }^{\forall} \Phi=(\check{\varrho}, \check{v}) \in \operatorname{SB}(\Gamma), \quad \bar{\partial}_{\theta} \check{\varrho} \text { is bounded on } \mathrm{SH}
$$

and

$$
{ }^{\forall} S \in \mathrm{SQ}(\Gamma), \quad \bar{\partial}_{\theta}\left[(z-\bar{z}-\theta \bar{\theta})^{2} \overline{S(z, \theta)}\right] \text { is bounded on } \mathrm{SH} .
$$

Note that if $\bar{\partial}_{\theta} \varrho$ is bounded on SH, $\chi[\Phi]$ satisfies that

$$
\chi[\Phi](z, \theta)=O\left(|\operatorname{Im} z|^{-1}\right)+\theta O\left(|\operatorname{Im} z|^{-2}\right),
$$

which is equivalent to the statement that $\bar{\partial}_{\theta}\left[(z-\bar{z}-\theta \bar{\theta})^{2} \overline{\chi[\Phi](z, \theta)}\right]$ is bounded.

\section{Appendix B. Residual Symmetry}

In this appendix, we take up the subjects which concern special properties of the $\mathrm{W}-\mathrm{Z}$ gauge. In the following, we assume $\Gamma$ to be a split super Fuchsian group. Let $\mathrm{SB}_{\mathrm{wz}}(\Gamma)$ denote the linear space consisting of $(-1,1)$ superconformal tensors $\mu$ such that $\bar{\partial}_{\theta} \mu=0$. The super Beltrami differentials in the $\mathrm{W}-\mathrm{Z}$ gauge form the bounded space of $\mathrm{SB}_{\mathrm{wZ}}(\Gamma)$ through the condition $(2.9 \mathrm{~b})$, which we denote by $\mathrm{SB}_{\mathrm{wZ}}(\Gamma)_{b}$.

The super Teichmüller space $\mathrm{ST}_{p}(\mathrm{SH} / \Gamma)$ is the quotient space of $\mathrm{SB}(\Gamma)_{b}$ by $\mathrm{sDiff}_{0}(\mathrm{SH} / \Gamma)$. We want to investigate the residual symmetry after the reduction from $\mathrm{SB}(\Gamma)_{b}$ to $\mathrm{SB}_{\mathrm{wz}}(\Gamma)_{b}$ is performed. Before looking at this, let us characterize the elements of $\mathrm{sDiff}_{0}(\mathrm{SH} / \Gamma)$. As in the ordinary Teichmüller theory, $\mathrm{sDiff}_{0}(\mathrm{SH} / \Gamma)$ is isomorphic to the group consisting of those $w$ and $I \circ w$ such that $w$ commutes with all elements of $\Gamma . \Gamma$ being split, then from Proposition 4.1 and 4.2, a quasisuperconformal mapping $w^{\Psi}=\left(w_{b}, w_{f}\right), \Psi \in \mathrm{SB}(\Gamma)_{b}$ commutes with every element of $\Gamma$ if and only if the following condition is satisfied:

$$
\begin{array}{cl}
{ }^{\forall} A \in \Gamma, & A_{b, 0} \circ w_{b \cdot 0}=w_{b \cdot 0} \circ A_{b \cdot 0}, \\
& \zeta \circ A_{b \cdot 0}=A_{f \cdot 0} \zeta,
\end{array}
$$


where $\zeta=w_{f \cdot 1} \circ w_{b \cdot 0}^{-1} \cdot{ }^{17}$ In this case of split $\Gamma$,(B.1a) is consistent with the boundary condition (3.8).

Now, let us consider for a given $\Psi=(-\bar{\theta} \mu, 0) \in \mathrm{SB}_{\mathrm{wz}}(\Gamma)_{b}$ an element $h$ in $\operatorname{sDiff}_{0}(\mathrm{SH} / \Gamma)$ such that $h^{*} \Psi$ also lives in $\mathrm{SB}_{\mathrm{wZ}}(\Gamma)_{b} \cdot{ }^{18}$ By making application of the formula (2.10), we see that ${ }^{19}$

$$
\begin{aligned}
& 0=\left(h^{*} v\right)_{\Psi} \leftrightarrow-D h_{b}+h_{f} D h_{f}=D \overline{h_{b}}(\mu \circ h), \\
& 0=\left(h^{*} \sigma\right)_{\Psi} \leftrightarrow-\bar{\partial}_{\theta} h_{b}+h_{f} \bar{\partial}_{\theta} h_{f}=\overline{\partial_{\theta} h_{b}}(\mu \circ h),
\end{aligned}
$$

and then

$$
\left(h^{*} \mu\right)_{\Psi}\left\{\partial_{z} h_{b}+h_{f} \partial_{z} h_{f}+\partial_{z} \overline{h_{b}}(\mu \circ h)\right\}=\bar{\partial}_{z} h_{b}+h_{f} \bar{\partial}_{z} h_{f}+\overline{\partial_{z} h_{b}}(\mu \circ h) .
$$

Through the Eqs. (B.2), the map $h$ has necessarily the $\mu$-dependence. The structure of the symmetry that remains under the $\mathrm{W}-\mathrm{Z}$ gauge may be best analyzed by considering $h$ close to the identity map. In references $[7,8]$, the symmetry algebra that acts on $\mathrm{SB}_{\mathrm{wz}}(\Gamma)_{b}$ has been investigated in the form of BRS transformations, introducing the reparametrization ghosts (which are made to turn from vector fields). For the sake of completeness and for clarifying the original geometrical setting, we describe it below. We set

$$
h(z, \theta)=(z+\varepsilon v(z, \theta), \theta+\varepsilon \zeta(z, \theta))
$$

with $\varepsilon$ being infinitesimal, and define a vector field on $\mathrm{SH} / \Gamma$ by

$$
\mathscr{V}=V \partial_{z}+\zeta D+\text { c.c. }
$$

where $V(z, \theta)=v(z, \theta)+\theta \zeta(z, \theta)$.

Provided $h \in \mathrm{SDiff}_{0}(\mathrm{SH} / \Gamma), V$ and $\zeta-\frac{1}{2} D V$ are $(-1,0)$ and $\left(-\frac{1}{2}, 0\right)$ superconformal tensors, respectively (see Appendix A). The infinitesimal forms of (B.2) and (B.3) are given by

$$
\begin{gathered}
\delta \Psi=(\delta \varrho, \delta v)=\mathscr{L}_{\mathscr{V}} \Psi, \\
0=\delta v=2 \zeta-(D V+\mu D \bar{v}), \\
0=\delta \sigma=-\bar{\partial}_{\theta}(V+\mu \bar{v}), \\
\delta \mu=\left[\bar{\partial}_{z}-\mu \partial_{z}+\left(\partial_{z} \mu\right)\right](V+\mu \bar{v})+\zeta D \mu .
\end{gathered}
$$

Here and below we omit the infinitesimal parameter $\varepsilon$. Equations (B.4) consist of six equations for the component fields of $V$ and $\zeta$ which leave their first components $\left.V\right|_{\theta=\bar{\theta}=0}=v_{0},\left.\zeta\right|_{\theta=\bar{\theta}=0}=\zeta_{1}$ free and make the other six components expressed in terms of $v_{0}$ and $\zeta_{1}$. Explicitly, (B.4) and (B.5) are summarized into [7],

$$
\begin{aligned}
\delta \mu_{0}= & {\left[\bar{\partial}_{z}-\mu_{0} \partial_{z}+\left(\partial_{z} \mu_{0}\right)\right]\left(v_{0}+\mu_{0} \overline{v_{0}}\right)+\zeta_{1} \mu_{1}, } \\
\delta \mu_{1}= & {\left[\bar{\partial}_{z}-\mu_{0} \partial_{z}+\frac{1}{2}\left(\partial_{z} \mu_{0}\right)\right]\left(2 \zeta_{1}+\mu_{1} \overline{v_{0}}\right) } \\
& +\left[\left(\partial_{z} \mu_{1}\right)-\frac{1}{2} \mu_{1} \partial_{z}\right]\left(v_{0}+\mu_{0} \overline{v_{0}}\right) .
\end{aligned}
$$

\footnotetext{
${ }^{17} w_{b .0}^{-1}$ is the inverse map of $w_{b \cdot 0}$ and not $\left(w^{-1}\right)_{b \cdot 0}$

18 In practice, the formulas (B.2) and (B.3) are valid for any quasisuperconformal map $h$ in the sense that $h^{*}$ preserves the $\mathrm{W}-\mathrm{Z}$ gauge condition

${ }^{19}$ Here we write the $v$-component of $h^{*} \Psi$, for example, as $\left(h^{*} v\right)_{\Psi}$
} 
The gauge freedoms concerning $v_{0}$ and $\zeta_{1}$ correspond to the ordinary diffeomorphism and local supersymmetry. Let $\mathscr{V}_{(1)}$ and $\mathscr{V}_{(2)}$ be the vector fields which satisfy (B.4), and set $\mathscr{V}_{(1)}=\mathscr{V}$,

$$
\mathscr{V}_{(2)}=\mathscr{W}=W \partial_{z}+\xi D+\text { c.c. },
$$

and

$$
\mathscr{V}_{(12)}=[\mathscr{V}, \mathscr{W}]=V_{(12)} \partial_{z}+\zeta_{(12)} D+\text { c.c. } .
$$

Then

$$
\begin{aligned}
V_{(12)} & =\mathscr{V}(W)-\mathscr{W}(V)-2 \zeta \xi, \\
\zeta_{(12)} & =\mathscr{V}(\xi)-\mathscr{W}(\zeta) .
\end{aligned}
$$

If we ignore the $\mu$-dependences of $\mathscr{V}_{(i)}$ by mistake, it might be viewed that the infinitesimal variations of the super Beltrami differentials form a Lie algebra of the ordinary super vector fields:

$$
\left[\delta_{1}, \delta_{2}\right] \Psi=-\mathscr{L}_{\mho_{12}} \Psi
$$

which is checked by the computation (ignoring the $\mu$-dependence of $\mathscr{V}_{(i)}$ ). Correctly, the $\mu$-dependence of $\mathscr{V}_{(i)}$ changes the result from above to

$$
\left[\delta_{1}, \delta_{2}\right] \Psi=-\mathscr{L}_{\tilde{r}_{12}} \Psi,
$$

where

$$
\tilde{\mathscr{V}}_{12}=\mathscr{V}_{(12)}+\left(\mathscr{L}_{W} \mu\right)_{\Psi} \cdot \frac{\partial}{\partial \mu} \mathscr{V}-\left(\mathscr{L}_{\mathscr{V}} \mu\right)_{\Psi} \cdot \frac{\partial}{\partial \mu} \mathscr{W} .
$$

To see $\widetilde{\mathscr{V}}_{12}$ satisfy (B.4), it suffices to show that

$$
\begin{aligned}
& \left(\mathscr{L}_{\tilde{V}_{12}} v\right)_{\Psi}=-\left.\left[\delta_{1}, \delta_{2}\right] v\right|_{\Psi}=0, \\
& \left(\mathscr{L}_{\tilde{V}_{12}} \sigma\right)_{\Psi}=-\left.\left[\delta_{1}, \delta_{2}\right] \sigma\right|_{\Psi}=0,
\end{aligned}
$$

which are apparent by regarding (B.4) as identity equations with respect to $\mu$. Therefore the algebra is closed on $\operatorname{SB}_{\mathrm{wz}}(\Gamma)_{b}$. The structure relation can be read from

$$
\begin{aligned}
&\left.\tilde{V}_{(12)}\right|_{\theta=\bar{\theta}=0}=\left.V_{(12)}\right|_{\theta=\bar{\theta}=0} \\
&= v_{0} \partial_{z} w_{0}+\overline{v_{0} \partial_{z}} w_{0}+\frac{\zeta_{1} \xi_{1}-\mu_{0} \overline{\zeta_{1} \xi_{1}}}{1-\mu_{0} \overline{\mu_{0}}}-\left(v_{0}, \zeta_{1} \leftrightarrow w_{0}, \xi_{1}\right), \\
& \tilde{\zeta}_{\left.(12)\right|_{\theta=\bar{\theta}=0}=} \zeta_{\left.(12)\right|_{\theta=\bar{\theta}}=0} \\
&=v_{0} \partial_{z} \xi_{0}+\overline{v_{0} \partial_{z}} \xi_{0}+\frac{1}{2} \zeta_{1}\left(\partial_{z} w_{0}+\mu_{0} \partial_{z} \overline{w_{0}}\right) \\
&+\frac{1}{2} \frac{\mu_{1}\left(\overline{\mu_{0}} \zeta_{1} \xi_{1}-\overline{\zeta_{1} \xi_{1}}\right)}{1-\mu_{0} \overline{\mu_{0}}}-\left(v_{0}, \zeta_{1} \leftrightarrow w_{0}, \xi_{1}\right) .
\end{aligned}
$$

This shows the $\mu$-dependence of the Lie algebra, which is familiar in supergravity as noted in $[7,8]$. 


\section{Appendix C. Supercomplex Structure of the Super Teichmüller Space}

The super Teichmüller space $\mathrm{ST}_{p}$ has a supercomplex structure entwined naturally with the superconformal structures on SRS's. In this appendix, we describe briefly the infintesimal approach to this problem [20]. This gives a rigorous treatment of the same subject that was studied in [23]. Another approach through the generalization of the Bers' embedding exist $[11,15]$. In [15], the global existence of the Bers' embedding for $\mathrm{ST}_{p}$ was shown.

Locally, $\mathrm{ST}_{p}$ is described as deformations from the split family of SRS's. The local coordinate systems around split SRS's are patched together to form an atlas of $\mathrm{ST}_{p}$. The super Beltrami differentials in the $\mathrm{W}-\mathrm{Z}$ gauge supply a class of natural coordinate systems which are the super-analogue of the Bers' coordinates [24], in a neighborhood of the initial point $\mathrm{SH} / \Gamma{ }^{20}$ First, we rewrite Proposition 2.4 to see the transformation from $\mathrm{SB}_{\mathrm{wz}}\left(\Gamma_{0}\right)_{b}$ to $\mathrm{SB}_{\mathrm{wz}}\left(\Gamma_{1}\right)_{b}$, supposing that SRS's $\Sigma_{0}$ $\left(=\mathrm{SH} / \Gamma_{0}\right)$ and $\Sigma_{1}\left(=\mathrm{SH} / \Gamma_{1}\right)$ are split ones. The transformation law becomes much simpler in this case. We set the quasisuperconformal maps $h: \Sigma_{0} \rightarrow \Sigma_{1}, g: \Sigma_{0} \rightarrow \Sigma_{2}$, and $w=g \circ h^{-1}: \Sigma_{1} \rightarrow \Sigma_{2}$ as before and write their associated super Beltrami differentials as $\Psi_{h}, \Psi_{g}$, and $\Psi_{w}$, respectively. The splitness of $\Sigma_{1}$ is reflected on the map $h$ in a obvious way as is shown by the following proposition.

Proposition C.1. If $\tilde{\mu}_{1}\left(\Psi_{h}\right)=0$, then $\Sigma_{1}$ becomes necessarily split. Conversely, if $\Sigma_{1}$ is a split SRS, then we can always set $\tilde{\mu}_{1}\left(\Psi_{h}\right)=0$.

Proof. If $\tilde{\mu}_{1}\left(\Psi_{h}\right)=0,(3.3 \mathrm{~b})$ gives the ordinary Beltrami equation for $h_{f \cdot 1}$. The boundary condition (3.8) means that $\tilde{\mu}_{1}\left(\Psi_{h}\right)=0$ is equivalent to $h_{f \cdot 1}=0$. Then, from (4.5b), $A_{f \cdot{ }_{1}}^{\Psi_{h}}=A_{b \cdot{ }_{1}}^{\Psi_{h}}=0$ follows. Conversely, if $\Sigma_{1}$ is split, we have from (4.5),

$$
\begin{gathered}
A_{b \cdot 0}^{\Psi_{h} \circ h_{b \cdot 0}}=h_{b \cdot 0} \circ A_{b \cdot 0}, \\
\zeta \circ A_{b \cdot 0}^{\Psi_{h}}=A_{f \cdot 0}^{\Psi_{h}} \zeta \quad \text { with } \zeta=h_{f \cdot 1} \circ h_{b \cdot 0}^{-1} .
\end{gathered}
$$

Since $A_{b \cdot 0}^{\Psi_{h}}$ is determined only through $h_{b .0}$, we are free to set $\zeta=0$ (that is to set $\tilde{\mu}_{1}=0$ ), being $h_{b \cdot 0}$ unchanged. Q.E.D.

What we are thinking about is to find the corresponding $\Psi_{w}$ in $\mathrm{SB}_{\mathrm{wZ}}\left(\Gamma_{1}\right)_{b}$ for a given $\Psi_{g}=\left(-\bar{\theta} \mu_{g}, 0\right) \in \mathrm{SB}_{\mathrm{WZ}}\left(\Gamma_{0}\right)_{b}$. Due to Proposition 4.1 and C.1, we can set $h$ to have the special form

$$
h(z, \theta)=\left(h_{b \cdot 0}, \theta k(z)\right) .
$$

Remember that $k(z)$ is irrelevant to the choice of $\Sigma_{1}$, and hence is allowed to be an arbitrary non-vanishing scalar function. Then, from (2.10), $\Psi_{w}$ takes the form $(-\bar{\theta} \mu, v)$, the expression of which is given by

$$
\begin{aligned}
& \mu \circ h=\frac{\partial_{z} h_{b .0}}{\partial_{z} h_{b .0}} \frac{\mu_{g}-\overline{\mu_{h}}}{1-\mu_{g} \overline{\mu_{h}}}, \\
& v \circ h=\frac{\theta}{k}\left\{\partial_{z} h_{b \cdot 0} \frac{1-\left|\mu_{h}\right|^{2}}{1-\mu_{g} \overline{\mu_{h}}}-k^{2}\right\},
\end{aligned}
$$

where $\mu_{h}=\frac{\bar{\partial}_{z} h_{b \cdot 0}}{\partial_{z} h_{b} \cdot 0}$.

${ }^{20}$ It is not essential for the supercomplex structure of $\mathrm{ST}_{p}$ to take the $\mathrm{W}-\mathrm{Z}$ gauge, but it simplifies the description 
Note that (C.2a) is the same form as is known for the ordinary Beltrami differentials aside from $\mu$ and $\mu_{g}$ being superfields here. By choosing the function $k(z)$ to vanish $v$ in (C.2b), we get $\Psi_{w}$ in $\operatorname{SB}_{\mathrm{wz}}\left(\Gamma_{1}\right)_{b}$.

The super-analogue of the Bers' coordinates are introduced as follows. As was shown in Appendix A, $\mathrm{SB}(\Gamma) / \mathrm{N}(\Gamma)$ is isomorphic to $\mathrm{SQ}(\Gamma)$ of which the dimensions are well known as

$$
\operatorname{dim}_{\mathbb{C}} \mathrm{SQ}(\Gamma)=(3 p-3 \mid 2 p-2) .
$$

We choose a relative even basis $\left\{\mu_{0}^{(i)}\right\}, i=1 \ldots 3 p-3$ and odd basis $\left\{\theta \mu_{1}^{(a)}\right\}$, $a=1 \ldots 2 p-2$ of $\mathrm{SB}_{\mathrm{wz}}(\Gamma)$ modulo $\mathrm{N}(\Gamma)$. Let $\tau=(t, \zeta)=\left(t_{1}, \ldots, t_{3 p-3}, \zeta_{1}, \ldots, \zeta_{2 p-2}\right)$ be a complex vector of the $(3 p-3 \mid 2 p-2)$-dim linear superspace. We set

$$
\mu(\tau)=\sum_{i=1}^{3 p-3} \mu_{0}^{(i)} t_{i}+\theta \sum_{a=1}^{2 p-2} \mu_{1}^{(a)} \zeta_{a}
$$

and consider the mapping $\tau \rightarrow \vartheta^{(-\bar{\theta} \mu(\tau), 0)}$. The following statements (a), (b) can be proved in just the same way with the ordinary non-super case.

(a) The complex vector $\tau$ serves the local coordinates of $\mathrm{ST}_{p}$ in a neighborhood of the initial point $\mathrm{SH} / \Gamma$.

The construction can be repeated for arbitrary initial point $\mathrm{SH} / \Gamma_{\alpha}$ with $\Gamma_{\alpha}$ being split. The transition functions among overlapping coordinates are specified through the formula (C.2) and the coordinate functions $\left\{\tau_{\alpha}(\mu)\right\}$ each defined near $\mu=0$ in $\operatorname{SB}_{\mathrm{wz}}\left(\Gamma_{\alpha}\right)_{b}$.

(b) These coordinate transformations are super-analytic in $\tau_{(\alpha)}$ 's and hence define a supercomplex structure on $\mathrm{ST}_{p}$.

A novel point in the super case is that because of Corollary 3.3, there still remains $\mathbb{Z}_{2}$ symmetry, which tells $(t, \zeta)$ and $(t,-\zeta)$ are the same point in $\mathrm{ST}_{p}$. In this sense, $\mathrm{ST}_{p}$ is a super orbifold. This fact was noted first in the approach of Bers' embedding [11].

Recently the Weil-Petersson Kähler form on $\mathrm{ST}_{p}$ was investigated, using the local coordinate systems which were the super-extension of those introduced by Fricke and Klein, and it was shown that the fundamental 2-form is closed [16]. It will be possible to analyze the Kähler structure of $\mathrm{ST}_{p}$ by extending the Ahlfors' work [24] in which was first studied the subject of non-super case.

Acknowledgements. The author would like to thank Y. Yasui for discussions and valuable comments.

\section{References}

1. D'Hoker, E., Phong, D.H.: The geometry of string perturbation theory. Rev. Mod. Phys. 60, 917-1065 (1988); Superstrings, super Riemann surfaces, and supermoduli space. Proceedings to the Rome conference on Strings and Mathematics. Columbia-UCLA/89/TEP/32 preprint (1989);

Nelson, P.: Lecture on supermanifolds and strings. Proceedings of T.A.S.I., Brown University 1988 ; and see references therein

2. Friedan, D.: Notes on string theory and two-dimensional conformal field theory. In: Green, M., Gross, D. (eds.) Unified string theories. Singapore: World Scientific 1986

3. Rabin, J., Freunt, P.: Supertori are algebraic curves. Commun. Math. Phys. 114, 131-145 (1988)

Rabin, J., Topiwala, P.: Super Riemann surfaces are algebraic curves; Super-GAGA and moduli of super Riemann surfaces. University of Chicago, preprint (1988) 
4. D'Hoker, E., Phong, D.H.: Superholomorphic anomalies and supermoduli space. Nucl. Phys. B 292, 317-329 (1987)

5. Giddings, S.B., Nelson, P.: The geometry of super Riemann surfaces. Commun. Math. Phys. 116, 607-634 (1988); Torsion constraints and super Riemann surfaces. Phys. Rev. Lett. 59, 2619-2622 (1987)

6. LeBrun, C., Rothstein, M.: Moduli of super Riemann surfaces. Commun. Math. Phys. 117, 159-176 (1988)

Falqui, G., Reina, C.: A note on the global structure of supermoduli spaces. Commun. Math. Phys. 128, 247-261 (1990)

7. Baulieu, L., Bellon, M., Grimm, R.: Factorization of the BRST algebra on superconformal structures. Nucl. Phys. B 321, 697-716 (1989)

Grimm, R.: Left-right decomposition of two-dimensional superspace geometry and its BRS structure. Ann. Phys. 200, 49-100 (1990)

8. Delduc, F., Gieres, F.: Beltrami differentials, conformal models and their supersymmetric generalizations. Class. Quantum Grav. 7, 1907-1952 (1990)

9. Grisaru, M.T., Xu, R.-M.: Quantum supergravities in two-dimensions. Phys. Lett. B 205, 486-492 (1988);

Polyakov, A.M., Zamolodchikov, A.B.: Fractal structure of two-dimensional supergravity. Mod. Phys. Lett. A 3, 1213-1219 (1988)

Grundberg, J., Nakayama, R.: On 2D (super) gravity in the light-cone gauge. Mod. Phys. Lett. A 4, 55-60 (1989)

10. Howe, P.S.: Super Weyl transformations in two dimensions. J. Phys. A 12, 393-402 (1979)

11. Crane, L., Rabin, J.: Super Riemann surfaces: Uniformization and Teichmüller theory. Commun. Math. Phys. 113, 601-623 (1988)

12. Rabin, J.: Teichmüller deformations of super Riemann surfaces. Phys. Lett. B 190, 40-46 (1987)

13. Uehara, S., Yasui, Y.: Super Beltrami differentials via 2D supergravity. Phys. Lett. 217, 479-484 (1989)

14. Hodgkin, L.: On metric and super-Riemann surfaces. Lett. Math. Phys. 14, 177-184 (1987); A direct calculation of super-Teichmüller space. Lett. Math. Phys. 14, 47-53 (1987)

15. Hodgkin, L.: Super-Beltrami differentials. Class. Quantum Grav. 6, 1725-1738 (1989)

16. Uehara, S., Yasui, Y.: The Weil-Petersson Kähler form on the super-Teichmüller space. Phys. Lett. B 250, 72-78 (1990); On the symplectic geometry of the super Teichmüller space. Kyoto university preprint YITP/U-90-16 (1990)

17. Batchelor, M.: Graded manifolds and supermanifolds. In: H. J. Seifert et al. (eds.) Mathematical aspects of superspace. Amsterdam: Reidel 1984

18. Rothstein, M.J.: The axioms of supermanifolds and a new structure arising from them. Trans. Am. Math. Soc. 297, 159-180 (1986)

19. Earle, C.J.: Teichmüller theory. In: Harvey, W.J. (ed.) Discrete groups and automorphic forms. New York: Academic Press 1977

20. Ahlfors, L.V.: Lectures on quasiconformal mappings. New York: Van Nostrand 1966

21. Batchelor, M.: The structure of supermanifolds. Trans. Am. Math. Soc. 253, 329-338 (1979) Gawedzki, K.: Supersymmetries - mathematics of supergeometry. Ann. Inst. Henri Poincaré A 27, 335-366 (1977)

22. Ahlfors, L.V., Bers, L.: Riemann's mapping theorem for variable metrics. Ann. Math. 72, 385-404 (1960)

23. Nelson, P.: Holomorphic coordinates for supermoduli space. Commun. Math. Phys. 115, $167-175$ (1988)

24. Ahlfors, L.V.: Some remarks on Teichmüller's space of Riemann surfaces. Ann. Math. 74, 171-191 (1961); Curvature properties of Teichmüller's space. J. Analyse Math. 9, 161-176 (1961) 
